November 26, 2021

\title{
Evolution of Human-specific Alleles Protecting Cognitive Function of Grandmothers
}

\author{
Sudeshna Saha ${ }^{1}$, Naazneen $\mathrm{Khan}^{1}$, Troy $\mathrm{Comi}^{2}$, Andrea Verhagen ${ }^{1}$, Aniruddha Sasmal ${ }^{1}$,
} Sandra Diaz ${ }^{1}$, Hai Yu ${ }^{3}$, Xi Chen ${ }^{3}$, Joshua M. Akey², Martin Frank ${ }^{4}$,

10 1. Departments of Medicine, Pathology, Anthropology and Cellular and Molecular Medicine, 11 Center for Academic Research and Training in Anthropogeny and Glycobiology Research and

12 Training Center, University of California San Diego, San Diego, California 92093, USA

13 2. Department of Genetics, Princeton University, Princeton, New Jersey 08544, USA

14 3. Department of Chemistry, University of California Davis, Davis, California 95616, USA

15 4. Biognos AB, Gothenburg, SE-402 74, Sweden

18 *Address correspondence to Ajit Varki: a1varki@ucsd.edu or Pascal Gagneux:

19 pgagneux@ucsd.edu. 


\section{Summary (250 Words)}

27 Late-onset Alzheimer's Disease (LOAD) pathology is rare in our closest living evolutionary

28 relatives (chimpanzees), which also express much lower microglial levels of CD33(Siglec-3)-a

29 myelomonocytic receptor inhibiting innate immune reactivity by extracellular V-set domain 30 recognition of sialic acid(Sia)-containing "self-associated molecular patterns" (SAMPs). We 31 earlier showed that $\mathrm{V}$-set domain-deficient CD33-variant allele, protective against LOAD, is 32 derived and specific to hominin-lineage. We now report that $C D 33$ also harbors multiple hominin33 specific $\mathrm{V}$-set domain mutations and explore selection forces that may have favored such genomic 34 changes. $N$-glycolylneuraminic acid (Neu5Gc), the preferred Sia-ligand of ancestral CD33 is 35 absent in humans, due to hominin-specific, fixed loss-of-function mutation in CMAH, which 36 generates CMP-Neu5Gc from its precursor, CMP- $N$-acetylneuraminic acid (Neu5Ac). Extensive 37 mutational analysis and MD-simulations indicate that fixed change in amino acid 21 of hominin 38 V-set domain and conformational changes related to His45 corrected for Neu5Gc-loss by 39 switching to Neu5Ac-recognition. Considering immune-evasive "molecular mimicry" of SAMPs 40 by pathogens, we found that human-specific pathogens Neisseria gonorrhoeae and Group B 41 Streptococcus (affecting fertility and fetuses/neonates respectively) selectively bind huCD33 and 42 this binding is significantly impacted by amino acid 21 modification. Alongside LOAD-protective 43 CD33 alleles, humans harbor additional, derived, population-universal, cognition-protective 44 variants absent in "great ape" genomes. Interestingly, 11 of 13 SNPs in these human genes 45 (including $C D 33$ ), that protect the cognitive health of elderly populations, are not shared by 46 genomes of archaic hominins: Neanderthals and Denisovans. Finally, we present a plausible 47 evolutionary scenario to compile, correlate and comprehend existing knowledge about huCD33 48 evolution and suggest that grandmothering emerged in humans.

Keywords (up to 10 words): Siglec3/CD33, pathogens, sialic acids, archaic genome, molecular dynamics simulation, phylogenetic analysis, menopause, grandmother. 


\section{Introduction}

54 In keeping with the fundamental importance of reproduction for the process of biological evolution

55 via natural selection, loss of fecundity generally coincides with the end of lifespan in almost all

56 species studied to date. Humans and certain toothed whales like orcas are so far the only mammals

57 known to manifest prolonged post-reproductive lifespans under naturalistic conditions [1-6]. One current explanation for such prolonged post-reproductive survival is late-life kin selection of grandmothers and other elderly caregivers of helpless young; apparently contrary to the concept

60 of "antagonistic pleiotropy", which posits that natural selection does not operate in late-life [7, 8].

61 An interesting exception is a human-specific derived allele of CD33 associated with direct or

62 indirect protection against late-onset Alzheimer's Disease (LOAD) [9]. Furthermore, we noted

63 that humans harbor additional examples of such derived, population-universal gene variants that

64 directly or indirectly impact late-life cognitive decline, which were not found in other "great ape"

65 genomes. This was considered as genomic evidence for the evolution of human postmenopausal

66 longevity [10]. Here we further explore the human-specific, derived alleles of genes that protect

67 against late-life cognitive decline, and ask when and how these emerged in hominins?

In vertebrates, glycan-binding proteins of the immunoglobulin (Ig) superfamily called sialic acid (Sia)-binding Ig-like lectins (Siglecs) form a major component of the immune system [11]. As the name indicates, Siglecs recognize Sias on cell surface or secreted glycoproteins and glycolipids. Siglec-3, commonly known as CD33, is the eponymous member of the rapidly evolving subgroup of Siglecs called CD33-related Siglecs (or CD33rSiglecs) [12, 13]. In contrast, other Siglecs (Siglecs 1, 2, 4 and 15) show evolutionary conservation [14]. CD33 is a type-I

74 transmembrane protein with an amino terminal Ig-like V-set domain followed by one Ig-like C2-

75 set domain proximal to the transmembrane region [13]. Its cytoplasmic tail contains 76 immunoregulatory signaling motifs called immunoreceptor tyrosine-based inhibitory motif 77 (ITIM)s, which upon ligand binding to the extracellular V-set domain, undergo phosphorylation 78 and recruit effector molecules like tyrosine phosphatases, SHP-1/2, which inhibit the cellular 79 immune response. Human CD33 (huCD33) binds $\alpha 2-3$ - and $\alpha 2-6$-linked $N$-acetylneuraminic acid 80 (Neu5Ac), the predominant Sia in humans, associated either with $\mathrm{N}$ - and O-glycosylated 81 molecules or sialylated glycolipids (gangliosides). HuCD33 undergoes alternative splicing, 82 resulting in two isoforms - full length CD33M containing the ligand binding $\mathrm{V}$-set domain and 83 truncated D2-CD33 (or CD33m) lacking this domain [15]. The elimination of the terminal V-set 
84 domain is mediated through differential splicing affected by two co-inherited single nucleotide 85 polymorphisms (SNPs) at positions rs3865444 in huCD33 promoter and rs12459419 located within exon 2 [16]. The two isoforms, CD33M and D2-CD33, differ not only in their molecular weights, but also in their cellular localization and functionality which are associated with Siainteracting V-set domain [9, 12, 17].

HuCD33 is extensively studied for its role in different immune responses, under both normal and pathophysiological conditions including cancers [16, 18, 18-21]. Furthermore, the microglial expression of CD33 is linked with neurological pathologies like LOAD. Incidence of LOAD has been strongly associated with varied expression of CD33 isoforms in the brain of

93 affected individuals [20, 21], where the LOAD-protective CD33 allele increases the ratio of D2-

94 CD33 isoform relative to CD33M. CD33 is reported in almost all vertebrates, including nonhuman primates $[6,14]$. While there is often high similarity in the sequence and overall genomic location, CD33 has undergone various species-specific changes. For example, murine CD33 which shows about 54\% identity with huCD33 V-set and 72\% identity with C2 domain, has markedly different Sia-binding and cellular expression patterns from human CD33 protein [22]. CD33 expression has greatly diverged in humans even in comparison to our closest living evolutionary relatives, the great apes. Examination of CD33 in peripheral blood showed significantly increased production of CD33M in human monocytes relative to those of chimpanzees [9]. Furthermore, the abundance of CD33 was also markedly higher in the human brain. Interestingly, although LOAD-associated

103 neurological pathologies, for example, buildup of $\mathrm{A} \beta$ proteins, hyperphosphorylated tau proteins 104 as neurofibrillary tangles, have been observed in aged nonhuman primate brains, AD has largely 105 been regarded as a uniquely human disease [23, 24]. Interspecies variations in CD33 have also 106 been studied in other apes like gorilla and bonobo, in comparison to huCD33 [25].

107 The presence of two physiologically significant isoforms, their distinct cellular localization and 108 association with uniquely human pathologies like LOAD have made huCD33 a target of much 109 evolutionary interest. The Sia-binding V-set domain of CD33rSiglecs including CD33 itself show 110 high sequence variability amongst different species, often making it difficult to identify their 111 orthologs. The selective pressure for this accelerated evolution of the V-set domains has been 112 attributed to evasion of infectious pathogens that exploit these human innate receptors. The 113 surfaces of each vertebrate cell are layered with tens to hundreds of million Sia-terminating 114 glycans, forming as "self-associated molecular patterns" (SAMPs), which prevent erroneous 
115 activation of innate immune responses against the body's own cells [26]. However, several human

116 pathogens e.g., Neisseria gonorrhoeae, Neisseria meningitidis, Haemophilus influenzae, E. coli

117 K1, Group B Streptococcus, and Trypanosoma cruzi cloak themselves with sialoglycans,

118 effectively mimicking host SAMPs, and thereby avoiding the immune response [27]. Conversely,

119 other infectious agents like influenza virus recognize SAMPs and utilize them as receptors to

120 initiate binding and subsequent infections [28]. CD33 has also been shown to interact with

121 Hepatitis B viral surface sialoglycans, thereby impacting its pathogenesis [29]. SAMPs and their

122 interacting partners, Siglecs (primarily the V-set domains) are therefore continually evolving to

123 maintain their distinctive "self-recognition" properties, while also avoiding exploitation by Sia-

124 cloaked pathogens and parasites - a powerful example of the "Red Queen Effect" [30].

125 In this work, with a focus on CD33, a post-reproductive cognitive health associated human protein,

126 we attempt to explore the evolutionary pressures that selected for unique changes in huCD33.

127 Using human-specific pathogens like Neisseria gonorrhoeae, Group B Streptococcus and E. coli

$128 \mathrm{~K} 1$, we demonstrate differential impact of these mutations on the bacterial interactions with

129 huCD33. We also determine the effect of these mutations on huCD33-sialoglycan binding and

130 identify that the amino acid at position 21 within the $\mathrm{V}$-set domain plays a critical role in Sia-

131 specificity of human and chimpanzee CD33. Furthermore, we extend our study to archaic hominin

132 genomes and show that the human-specific CD33 mutations (except the presence of truncated

133 isoform) are shared evolutionary changes of human, Neanderthal and Denisovan common

134 ancestor. We also expanded our analysis to include other human-specific derived genomic changes

135 associated with cognitive health of post-reproductive human grandmothers and other elderly

136 caregivers. Finally, we draw an evolutionary scenario to connect the current knowledge of CD33

137 sialoglycan recognition and pathogen engagement to propose a role for the infectious pathogens

138 as key selective agents in human-specific CD33 evolution, generating new alleles protective

139 against infections, that could secondarily have come under selection for their protective effects

140 against cognitive pathologies like LOAD.

Results

143 Sequences of human CD33 extracellular domains show many changes distinct from closely

144 related great apes. Previous investigations have identified unique properties of huCD33 that

145 influence the functionality of this molecule in humans. The presence of a huCD33 V-set truncated 
146 isoform as well as its overall expression difference in microglia has been associated with the

147 protection against the occurrence of neurological pathologies like LOAD in humans. Like other

148 CD33rSiglecs, CD33 immunomodulatory roles depend both on its ligand-interacting extracellular

149 domains and signaling motif-containing cytoplasmic tail. To gain a comprehensive understanding

150 of different CD33 domain variations, we compared the amino acid residues of full-length CD33

151 from human and related nonhuman primates including chimpanzee, gorilla and bonobo (Figure

152 1A). While the regions encoding the $\mathrm{C} 2$-set domain and cytoplasmic tail are highly conserved, the

153 amino acid residues within huCD33 V-set domain differ from their nonhuman counterparts in as

154 many as 10 positions. Since different amino acid residues in Sia-binding V-set domain could

155 potentially impact huCD33-sialoglycan interactions and subsequent downstream signaling

156 pathways, we further examined the overall frequency of these changes (Figure 1B). We analyzed

157 human sequences from the 1000 Genome database [31] and compared them with 44 gorilla, 59

158 chimpanzee and 10 bonobo sequences [32-34]. Most of these amino acid residues (except at

159 positions 66 and 148) are conserved in all the great apes and appeared to have changed only in the

160 human lineage. Interestingly, the amino acid residues at positions 66 and 148 in huCD33 are

161 isoleucine and leucine respectively, similar to CD33 of chimpanzee and bonobo. The

162 corresponding amino acids in its more distant evolutionary relative, gorilla, are phenylalanine

163 (Phe) (at position 66) and valine (at position 148). The presence of the same amino acid in human,

164 chimpanzee and bonobo at these positions suggests a more ancient occurrence of these two

165 changes, possibly prior to the divergence of chimpanzee about 6-8 million years ago (mya).

166 Previously it has been shown that the two linked SNPs, resulting in the splicing of the V-set

167 truncated isoform represent a derived evolutionary modification of the CD33 proteins in humans

168 and are absent in chimpanzees [9].

169 To further understand the selection pressure, we calculated the nonsynonymous to synonymous

170 substitution rate ratio (omega, $\omega=\mathrm{d}(\mathrm{N}) / \mathrm{d}(\mathrm{S})$ ) for the CD33 V-set domains of human and other

171 great apes. The omega value of CD33 V-set domain is greater than $>1(\omega=1.49)$ which reflects V-

172 set domain evolution under positive selection. Subsequently, we also analyzed the $\mathrm{Ka} / \mathrm{Ks}$ ratios of

173 exon 2 sequences in every species. Except for gorilla, the other two great apes (chimpanzee and

174 bonobo) showed $\mathrm{Ka} / \mathrm{Ks}$ ratios greater than one indicating that high $\mathrm{Ka} / \mathrm{Ks}$ ratio of exon 2 is not an

175 accidental event but an evolutionary phenomenon. Taken together, these results demonstrate that 
176 CD33 in humans has been rapidly evolving possibly under positive selection, distinct from its

177 orthologs in the great apes.

178 Archaic Neanderthal and Denisovan genomes share most human CD33 protein changes,

179 except for the SNPs for the LOAD-protective allele. Divergence of humans from other ancient

180 hominin lineage such as Neanderthals and Denisovans has been estimated to date back 181 approximately 0.5 mya [35]. Although full length CD33 itself is an ancient molecule, we noted 182 that the AD-protective CD33 truncated isoform is recently derived in humans, postdating our 183 divergence from Neanderthals and Denisovans [9]. Since huCD33 extracellular domains showed 184 high accumulation of changes compared to the great apes, we wanted to determine if these changes 185 were present in the common ancestor of the hominin lineage. We therefore compared CD33 protein 186 coding sequences from 6 Neanderthal and 2 Denisovan archaic genomes obtained from the Max 187 Planck Institute for Evolutionary Anthropology [36] (http://cdna.eva.mpg.de) with the 188 corresponding human sequences of the 1000 Genome database (Figure 1B). Interestingly, all the 189 amino acid residues in huCD33 that are different from the great apes are present in the ancient 190 genomes, suggesting their occurrence in a common ancestor. These observations thus suggest that 191 the complete loss of Sia-binding V-set domain is the latest evolutionary modification of huCD33, 192 likely succeeding the individual amino acid changes within its extracellular domain.

193 A single amino acid change facilitated CD33 engagement to the uniquely human pathogen

194 Neisseria gonorrhoeae. In addition to microglial expression in the brain, CD33 is also present on 195 tissue macrophages and peripheral blood monocytes [9]. These cells are important components of 196 innate immune responses throughout the body, including the reproductive tract. The human female 197 reproductive tract is also a unique niche for the microbiome, which can be invaded by important 198 pathogens like Neisseria gonorrhoeae $(\mathrm{Ng}) . \mathrm{Ng}$ is a uniquely human infectious agent, responsible 199 for the second most prevalent, sexually transmitted infection causative for the disease gonorrhea 200 in human populations. Gonorrhea affects both males and females and if untreated, can have 201 detrimental effects on reproductive health [37]. We have previously shown that $\mathrm{Ng}$ interacts with 202 human CD33 but not the chimpanzee ortholog [38]. The bacterium is incapable of endogenous 203 Neu5Ac synthesis, but instead scavenges the molecule from its host [39, 40]. Once inside the 204 female reproductive tract, $\mathrm{Ng}$ utilizes the host sugar nucleotide CMP-Neu5Ac from its 205 microenvironment to transfer Neu5Ac onto its own bacterial lipooligosaccharide. Sialylated $\mathrm{Ng}$ 206 then successfully interacts with several human Siglecs including 3 (CD33), 5, 9, 11, 14 and 16 
207 [38]. However, unlike other Siglec interactions, Ng binding to CD33 appears to be entirely Sia208 dependent. Interestingly, of all the Neisseria species currently known, only $\mathrm{Ng}$ and Neisseria 209 meningitidis are pathogenic to humans and both are thought to be evolutionarily young compared 210 to others [41]. Since reproductive health/success of an organism is the key determinant of 211 Darwinian fitness, we hypothesized that highly infectious disease like gonorrhea could potentially 212 impact the evolution of humans, mediated through binding immune modulating proteins like 213 CD33.

214 To explore our hypothesis, we examined the binding of sialylated $\mathrm{Ng}$ to different recombinant 215 CD33 protein mutants, each containing the two extracellular domains with an amino acid residue 216 changed from human to chimpanzee at the corresponding positions identified in Figure 1B. 217 Fluorescently labelled $\mathrm{Ng}$ was allowed to interact with human recombinant Fc-chimeric constructs 218 of the CD33 proteins that were immobilized onto protein A-coated plates (Figure 2A). Sia219 dependence of the interaction was confirmed by comparing binding with bacteria grown in 220 presence and absence of CMP-Neu5Ac (Supplemental Figure S1A). We observed significant 221 reduction in bacterial binding to chimpanzee CD33 (chCD33) compared to human protein 222 containing both V-and C2- domains (Figure 2B). However, in the absence of the V-set domain in 223 the truncated form of huCD33 (CD33m), bacterial binding was lost. Except for the residue at 224 position 21, all the other amino acid alterations from human to chimpanzee CD33 maintained high 225 bacterial binding. In fact, changing the amino acid residues at positions 22, 65 (of the V-set 226 domain), 152, and 154 (of C2 domain) increased the binding significantly compared to wildtype 227 huCD33. In contrast, mutating the amino acid at position 21 from human to chimpanzee residue 228 completely abolished huCD33 binding of sialylated $\mathrm{Ng}$. Interestingly, mutating the chimpanzee 229 CD33 amino acid at position 21 to its corresponding human residue enabled $\mathrm{Ng}$ to now engage 230 chimpanzee CD33 (Figure 2C). Considering that $\mathrm{Ng}$ and its closest relative meningococcus are 231 both uniquely human pathogens thought to have evolved from commensal Neisseria [42], our data 232 suggest important implications of CD33 amino acid change at position 21 on Ng-huCD33 233 interaction and their mutual evolution.

234 Many amino acid changes in CD33 extracellular domains impact GBS engagement. While 235 the association of Neisseria with CD33 is a case of Sia-mediated interaction, there are other 236 examples of human pathogens that engage Siglecs in Sia-independent manner. One such example 237 is Group B Streptococcus (GBS) which has been widely studied for its various ways of engaging 
host Siglecs [43]. GBS is an encapsulated pathogen commonly associated with pneumonia, sepsis and meningitis in infants and neonates. It comprises nine serologically distinct groups (Ia, Ib and II -VIII), differing in their capsular sialoglycan structures, but all containing $\alpha 2-3$-linked terminal Neu5Ac. Certain GBS strains have been shown to bind human Siglecs 5 and 7 in a Sia-independent manner through cell wall anchored $\beta$-protein [44], whereas some Sia-dependent binding was observed for CD33 and Siglec-9. Human Siglec-9 binding is also thought to be partially Siaindependent. Interestingly, some GBS strains are also known to interact with nonhuman primate Siglecs, for example, Siglec-9 from chimpanzee [25]. Since infections by GBS mostly impact newborns and infants, we hypothesized that it could also play a role in overall Siglec evolution in humans. Similar to the Ng-CD33 binding assay (as in Figure 2A), we examined the interactions between the recombinant CD33 proteins and GBS group III strain, COHI (Figure 2D). While the bacteria bound strongly with full-length extracellular domains of huCD33, the binding was significantly reduced in the truncated human isoform $(\mathrm{CD} 33 \mathrm{~m})$ and the chimpanzee protein. Like $\mathrm{Ng}$, GBS COHI interaction was also markedly disrupted by amino acid changes at position 21. Additionally, changing the residues at positions 20 and 65 from human to chimpanzee significantly reduced the bacterial interaction with CD33. However, GBS COHI engagement with the CD33 mutants was not entirely Sia-dependent (Figure 2E). Using GBS COHI $\Delta n e u A$, a mutant strain

255 lacking its sialyltransferase enzyme (NeuA) and hence incapable of surface sialylation, we observed that about $50 \%$ of the bacterial binding to CD33 could be attributed to Sia-independent interactions. Interestingly, the CD33 binding profile of COHI was not uniform for the other

258 serogroups of GBS, for example GBS group Ia strain, A909 (Figure 2F). None of the amino acid 259 changes showed significant effects on CD33 interaction with GBS A909, relative to the wildtype 260 human protein. Even the truncated human CD33 isoform (CD33m) displayed similar binding 261 suggesting that the CD33 binding for A909 is primarily Sia-independent. Unlike $\mathrm{Ng}$ and GBS, we 262 did not observe any differential sialoglycan binding with E. coli K1, another uniquely human 263 pathogen of newborn infants, which contains Sia polymers on its surface (Supplemental Figure 264 S1B). Altogether, the data demonstrate the diverse nature of CD33-interactions in three major 265 pathogens and suggest an impact of uniquely human pathogens in the evolution of CD33 ligand266 binding domain.

267 Ancestral sialoglycan preference of CD33 is disrupted by amino acid change at position 21. 268 A key change in the evolution of humans was the loss of CMP-Neu5Ac hydroxylase (CMAH), the 
enzyme that converts CMP-Neu5Ac to CMP-Neu5Gc resulting in a primarily Neu5Ac-rich sialome in humans, unlike any other Old-World primates, which express both Neu5Ac and

271 Neu5Gc. This change is dated to 2-3 mya when human ancestors were evolving from ancestral 272 hominins. Since we observed numerous changes mainly in huCD33 V-set domain which is critical 273 in sialoglycan interaction and therefore important for its downstream signaling pathways, we 274 wanted to specifically understand the effect on CD33 sialoglycan interactions. We used a 275 microarray of chemoenzymatically synthesized glycans with defined structures, terminally capped 276 with either Neu5Ac or Neu5Gc in different glycosidic linkages and examined their relative 277 interactions with recombinant, soluble CD33 proteins (Figure 3). Human CD33 with V- and C2278 domains bound to both Neu5Ac and Neu5Gc-terminating sialoglycans and showed maximum 279 binding when the Sia was a2-6-linked to an underlying lactose or lactosamine glycan 280 (Supplemental Figure S2). Most of this binding was lost in the truncated huCD33 lacking the Sia281 binding V-set domain, indicating that the interactions are Sia-dependent. Conversely, the 282 chimpanzee protein (which is identical to the bonobo orthologs and differs by only two amino 283 acids from the gorilla) demonstrated strong preference towards Neu5Gc-terminating sialoglycans 284 and showed almost no binding for Neu5Ac-epitopes. Considering the varied sialoglycan profiles 285 of the two organisms, these distinct binding preferences of human and chimpanzee CD33 are 286 interesting and suggest functional implications of the evolutionary changes in their extracellular 287 domains.

288 Because the impact of amino acid residue at position 21 was most pronounced in both of our 289 bacterial-CD33 binding assays (Figure 2B and 2D), we next examined the influence of this change 290 on CD33-sialoglycan binding (Figure 3 and Supplemental Figure S2). Indeed, changing the amino 291 acid at position 21 completely altered Sia-epitope preference of CD33 for both human and 292 chimpanzee. The presence of human amino acid residue at position 21 enabled strong binding of 293 Neu5Ac-epitopes by chCD33, unlike its entirely Neu5Gc-preferring wildtype counterpart. On the 294 other hand, the chimpanzee amino acid at the same position in human CD33 abolished its Neu5Ac 295 binding. To determine if the Sia-binding changes are specific for position 21 and not an arbitrary 296 effect of any amino acid change in V-set domain, we also looked at the Sia-epitopes of position 20 297 amino acid substitutions. Unlike position 21, amino acid modifications at position 20 did not have 298 any major impact on the Sia-binding of CD33, which maintained the overall wildtype profile. 299 Interestingly, modifications at position 22 demonstrated Neu5Ac-prefered binding for huCD33, 
while chimpanzee amino acid residues at 65 and 66 of huCD33 almost abolished any sialoglycan binding. Altogether, the data emphasized the importance of different amino acid changes in huCD33 V-set domain for its sialoglycan binding and identified the amino acid at position 21 to be critical in the functionality of CD33 protein.

MD simulations provide structural insights for the differences in Sia-binding preference based on molecular dynamics simulations. A detailed analysis of several available crystal structures of huCD33 revealed that the V-set domain is dynamic. For example, the C-C' loop as well as the side chains of phenylalanine at position 21 (Phe21) and histidine at position 45 (His45) are resolved in two different conformations in PDB entry 5ihb (Supplemental Figure S3). Of all the amino acids that differ between human and chimpanzee, only the side chain of Phe 21 is in direct contact with a bound Neu5Ac residue in the crystal structures of huCD33 (through the methyl group at position 5). Based on the assumption that Neu5Gc binds to the same binding site as Neu5Ac, the change in binding preference from Neu5Gc (in chimpanzee) to Neu5Ac (in human) cannot be explained by a simple I21F mutation. Both amino acids have hydrophobic side chains that cannot establish favorable interactions with the polar glycolyl group. Consequently, there is probably a more complex reason for the shift of binding preference. Based on data derived from 47 molecular dynamics (MD) simulations covering an accumulated timescale of more than $100 \mu \mathrm{s}$ we conclude that in chCD33 His45 adopts mainly the 'up' conformation (Figure 4A), which allows favorable hydrogen bonding with the glycolyl group. MD simulations (as well as $\mathrm{x}$-ray crystallography) show that in huCD33 His45 can also exist in the 'up' conformation (Figures 4C and Supplemental Figure S4), which would be compatible with favorable Neu5Gc binding. However, when His45 is in the 'down' conformation Phe21 can stack partly with tyrosine (Tyr) at position 127 (Figure 4B) forming a small hydrophobic pocket, which allows the methyl group of

324 Neu5Ac to bind favorably. To demonstrate if the binding affinity difference between Neu5Ac and 325 Neu5Gc may be indeed correlated to the up/down conformational equilibrium of His45, we 326 performed a series of MD simulations of chCD33 on the microsecond timescale where 327 Neu5AcOMe or Neu5GcOMe molecules are present in the solution. The lifetimes of the 328 complexes spontaneously formed during the MD with Neu5Gc are on average much longer when 329 His45 is 'up' (Figure 4D top, Supplemental Figure S4). In contrast the lifetimes of the complexes spontaneously formed with Neu5Ac are much shorter independent of the conformational state of 
331 His45 (Figure 4D bottom), which would explain the lack of measurable binding affinity of Neu5Ac 332 to chCD33. In summary, our extensive MD simulations - including unbiased simulation of 333 carbohydrate binding and unbinding events - could provide a reasonable explanation for a change 334 in binding specificity that is likely to be caused by an alteration of the protein-ligand interaction 335 pattern remote from the mutated amino acid.

336 Human-specific polymorphisms in cognitive-health related genomic variants are present in

337 all human populations. In an earlier study we observed several genes, directly associated with 338 neurodegenerative diseases or correlated with aggravation of the cognitive decline in aged339 population, are derived alleles in humans [9]. Increasing evidence of correlation between cognitive 340 health and non-neurological, metabolic conditions, e.g., diabetes [45, 46] suggest that such derived 341 alleles could be important in the maintenance of cognitive health in human grandparents. Here, we 342 expanded this list of cognition-protective gene variants through literature and database 343 (https://alzoforum.org) searches [20, 47-58] to include additional gene variants, namely BINI, 344 ARID5B, PICALM, PILRA. Supplemental Table S1 describes the characteristics of 13 human 345 genes that are implicated in diseases including dementia, cardiovascular diseases (CVD), 346 hypertension and AD. While some of these physiological abnormalities like salt retention, 347 hypertension, diabetes, appear non-neurological, they have been associated with the aggravation 348 of the pathologies resulting in late-life cognitive decline [59]. Notably, the derived alleles are 349 common and found in globally diverse human populations, indicating that they predate the 350 common ancestor of modern humans (Supplemental Table S2).

351 SNPs associated with human-specific cognitive protective alleles are unusual in their absence

352 in the archaic hominin genomes. With the availability of genomes from extinct archaic hominins $353[36,60,61]$, a set of SNPs can be assessed as to whether their protective phenotypes arose recently 354 in the evolutionary history of anatomically modern humans. We previously showed many other 355 human-chimpanzee differences were shared with archaic hominins (Denisovan/Neanderthal) 356 genomes; for example, genomic changes in CD33rSiglecs [62]. To gain similar insights about the 357 evolutionary origin of these cognitive-protective loci, we analyzed the Neanderthal and Denisovan 358 reference genomes and compared them with modern human sequences. Analysis of the 1000 359 Genomes dataset shows the presence of protective alleles in human populations with variable 360 frequency (Table 1). Analysis of the available genomic data from Neanderthal and Denisovan 361 genomes showed that only two derived variants (rs2975760 and rs2588969; Table 1) are present 
in these archaic genomes, suggesting the remaining eleven derived, protective variants arose after

363 the divergence of modern and archaic hominins approximately 0.5 mya $[35,63]$. This is in striking

364 contrast to most human-chimpanzee genomic differences in which the archaic hominins are similar

365 to humans. In fact, majority of the Sia-related genes lack positive selection signatures and rather

366 show neutral evolution in the modern human lineage [64]. To more formally assess whether the

367 high frequency, global distribution, and recent origin observed for eleven of the thirteen SNPs is

368 unusual, we performed a resampling analysis of variants in the genome. Variants in the 1000

369 genomes dataset that met the following criteria were considered: 1) present in both Altai

370 Neanderthal and Denisovan minimal filters, 2) derived in at least one modern individual from non-

371 admixed African populations, 3) called in both archaic samples, and 4) have an ancestral allele

372 matching the reference or alternative allele. To eliminate any bias in the analysis and match the

373 allele frequency (AF) of these SNPs compared with that of any random SNPs, we first matched

374 our universe of SNPs to the 13 SNPs of interest by AF, \pm 2 derived haplotypes (Figure 5).

375 Resampling was then performed by drawing a SNP from each of the 13 matched sets and assessing

376 how many derived alleles were observed, resulting in a $p$-value $=0.08333 \pm 0.00003$. As a less

377 conservative estimate, directly sampling from the universe of SNPs and estimating the probability

378 of observing at most two derived SNPs and a mean allele frequency as large as the empirical

379 variants of interest produced a highly significant $p$-value $=0.00487$ (Supplemental Figure S5).

380 Repeating either analysis on the set of other Siglec-related SNPs indicates they are consistent with

381 a random draw from the genome [62]. Regardless of the individual limitations, taken together our

382 phylogenetic analyses demonstrate the unique patterns of allele frequencies in worldwide

383 populations distribution of these thirteen late-life cognitive decline linked SNPs (Figure 5 and

384 Supplemental Figure S5). Interestingly, co-inherited CD33 SNPs associated with the cognitive

385 health in LOAD are present only in modern human genomes [9]. A noteworthy example in our list

386 is the human gene encoding the protein, apolipoprotein E (APOE), involved in fat metabolism in

387 mammals. APOE gene exists in three allelic variants (E2, E3 and E4) where APOE4 is associated

388 with high risk of LOAD and other allele like APOE2 is protective against the cognitive decline in

389 elderly caregivers [65]. Interestingly the presence of APOE4 is also correlated with the protection

390 from severe diarrhea in children [66]. While conclusive determination of the positive selection of

391 these alleles in modern human requires further analysis, our data suggest that the evolutionary

392 origin of most of these cognitive-health protective changes followed the divergence of modern 
393 humans from archaic genomes. This is also supported by the presence of grandparents, uniquely

394 in humans. Regardless, the process of evolutionary emergence of each of these alleles is likely to

395 be distinct and deserves further investigation.

\section{Discussion}

398 Fossil evidence and genomic comparisons leave little doubt about the fact that our species evolved 399 from an African hominin. However, a detailed understanding of modern human origins is plagued 400 by numerous uncertainties, with regard to the identity of the ancestral lineage and precise 401 geographic locations. Evolution of modern humans was accompanied by many anatomical and 402 behavioral changes, but increasing evidence suggests it also included uniquely human-derived 403 modifications in the genome compared to the archaic genomes (Neanderthal/Denisovan) or the 404 genome of the great apes [9, 62]. Taken together with our previous study [9], we have identified 405 many such human-specific genes associated with cognitive health of grandmothers and other 406 human elders who are often involved in the caregiving of the young. These findings, which appear 407 paradoxical to the concept of senescence due to antagonistic pleiotropy, have lent much additional 408 support to the "Grandmother hypothesis" [1] bolstering the case for selection of human female 409 post-reproductive survival and the existence of grandmothers. Unlike in any other mammals 410 (except orcas and some other toothed whales), the occurrence of this prolonged post-reproductive 411 life span in humans has stirred scientific interest. While deciphering the precise evolutionary 412 course of any gene/protein is challenging and the proposed schemes/players are not entirely 413 verifiable, here we attempt to compile the current evolutionary and experimental findings of one 414 such protein associated with late-life cognitive-decline: CD33.

415 A ratio of high wildtype human CD33 and a low truncated isoform of CD33 have been implicated 416 in the progression of LOAD associated with the cognitive health of elderly population. In contrast, 417 LOAD is unknown in chimpanzees, although evidence of LOAD pathologies has been observed 418 in some chimpanzee brains. We found that human CD33, which is highly expressed in microglia 419 of the human but not chimpanzee brain, recognizes Neu5Ac - the predominant Sia synthesized in 420 humans - as self-associated molecular patterns (SAMPs). In contrast, our closest evolutionary 421 relative, the apes and other Old-World primates contain both Neu5Ac and Neu5Gc. We found that 422 the ancestral form of CD33 in chimpanzees and other great apes selectively recognizes only 423 Neu5Gc-glycans as SAMPs (Figure 3). Notably, Neu5Gc - the ligand recognized by chCD33 is 
424 rare in chimpanzee brain, and there is also significantly less chCD33 protein compared to CD33 425 in humans [9]. On the other hand, SNPs resulting in the truncated CD33 have only been observed 426 in the human genome and not any of the archaic or great ape genomes. We also find that the 427 truncated human CD33 does not interact with Sia (Figure 3). Taken together, these observations 428 suggest that full-length CD33-Sia interactions are stronger in human brain compared to 429 chimpanzee and the human-specific SNPs in CD33 resulting in the truncated protein abolish this 430 interaction. The question remains what could have possibly led to the selection of the truncated 431 isoform of human CD33 that does not interact with Sia. In this regard, CD33 on macrophages 432 plays crucial roles in different immune responses as well as during infections. Human CD33 has 433 also recently been shown to be involved in immunomodulation during infection with hepatitis B 434 virus [29]. Our previous and current data show that uniquely human pathogens like Neisseria and 435 GBS display Neu5Ac that is recognized as 'self' by human but not chimp CD33 [38]. In the current 436 work, we further found that the Sia-binding-domain-depleted, truncated human CD33 isoform 437 doesn't bind and thus escape exploitation by sialylated pathogens (Figure 2). This suggests that 438 this truncated CD33 may have been an adaptation to counter the CD33-exploiting, immune439 evasive behavior of pathogens like Neisseria and GBS.

440 Taking together all currently available experimental data (including this study) we attempt to draw 441 a plausible evolutionary scenario for CD33 protein evolution in humans and present in the context 442 of relevant evolutionary events (Figure 6). We hypothesize that the scarcity of the strongly 443 preferred Neu5Gc ligand of ancestral CD33 in the brains of chimpanzee (and other great apes) was 444 associated with low microglial expression. Subsequent hominin loss of CMAH (i.e., complete loss 445 of Neu5Gc ligand) could then have selected for the upregulation of CD33 levels perhaps to 446 compensate for the loss of ligands, a change to Neu5Ac-binding preference, and functional 447 recruitment of CD33 to human microglia. Alongside the microglial CD33, the corresponding 448 changes in the tissue macrophage proteins might have facilitated the emergence of Neu5Ac-coated 449 pathogens (for example, N. gonorrhoeae and Group B Streptococcus) that evolved "molecular 450 mimicry" of Neu5Ac-SAMP ligands to manipulate the immune response. Appearance of the 451 truncated isoform lacking the ligand-binding domain (CD33m), then probably allowed CD33 to 452 escape the immune evasion by these sialylated pathogens (Figure 2). This selection pressure to 453 stop manipulation by sialylated pathogens could have also altered splicing towards a higher level 454 of truncated CD33, which also gets diverted to peroxisomes [12]. While the significance of this 
diversion is unclear, decrease of full-length CD33 would facilitate escape from Neu5Ac-coated, CD33-engaging pathogens. Finally, sometime during the last 1 million years, increased brain size presumably selected for early, short interbirth interval in human, which might have resulted in more helpless young, requiring cooperative breeding and caregiving. However, the value of postmenopausal grandmothers and other elderly caregivers would then have been blunted by the appearance of LOAD. The synthesis of the truncated isoform of CD33 protects from Neisseria during reproductive age and a higher ratio of truncated to full-length isoforms correlates to decrease of LOAD in grandmothers. However, a small amount of the full-length isoform remains, likely to downregulate hyper-inflammation that might arise during prolonged absence of SAMPrecognition. Notably when an elderly caregiver gets LOAD, not only are the evolutionary benefits of the individual lost, but this also presents an increased burden to care for that elder individual. Altogether under this proposed scenario, the current state in the evolution of human CD33 protein represents a trade-off between the evolutionary response to exploitation by pathogens in early life and cognitive maintenance in post-reproductive late life.

A similar evolutionary scenario appears to underly the case of the human $A P O E$ gene where variants include both risk alleles (APOE4) and protective alleles, (APOE2, and APOE3) for CVD and LOAD [65]. In this instance, the ancestral APOE4 allele is associated with increased risks of loss of cognitive functions and the derived alleles may serve to protect the cognition of the elderly caregivers. Interestingly the APOE4 allele is also correlated with the protection from severe diarrhea in early years of life [66]. Given these examples like APOE and CD33, it remains to be seen how widespread this evolutionary pattern is wherein variants conveying survival advantages in early life coexist with other variants that protect cognition late in life.

\section{Acknowledgments}

479 We are very thankful to Dr Sanjay Ram (University of Massachusetts, Worcester) and Dr. Victor 480 Nizet (University of California, San Diego) for the bacterial strains that have greatly benefited the 481 project. This work was supported by NIH grant R01GM32373 and Cure Alzheimer's Fund grant 482 (to A.V.).

\section{Author Contribution}

484 Experimentation (S.S., N.K., T.C., A.V., A.S., S.D., M.F.), Data analysis (S.S., N.K., T.C., 485 A.V., A.S., S.D., J.M.A., M. F., P. G., A.V.), Critical reagents (H.Y., X.C.), Original draft (S.S., 
486 P.G., A.V.), Writing (S.S., N.K., T.C., A.V., A.S., X.C., J.M.A., M.F., P.G., A.V.), Overall 487 supervision (J.M.A., P.G., A.V.), Funding acquisition (A.V.).

489 The data for the resampling analysis is available at Code Ocean. 
A

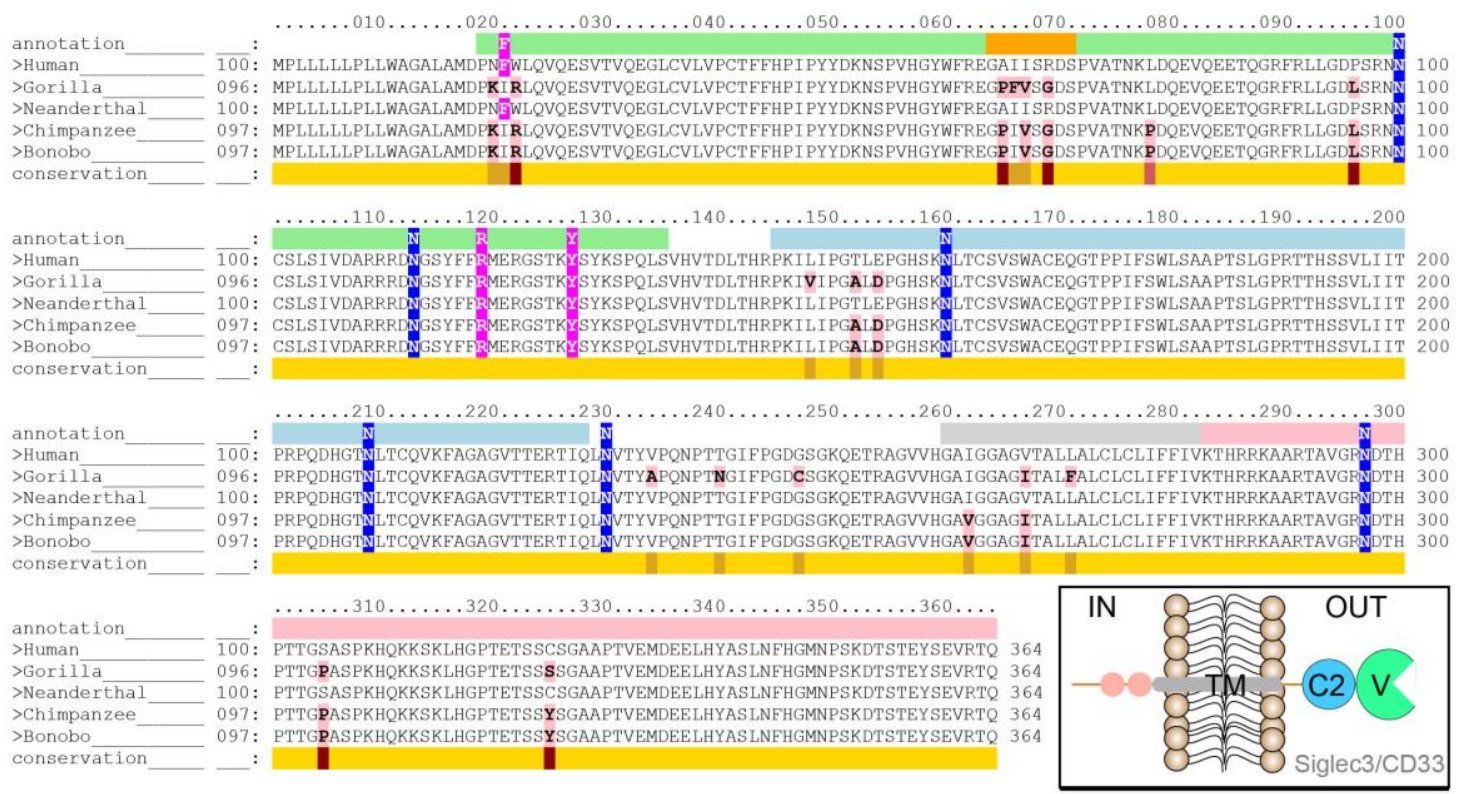

B

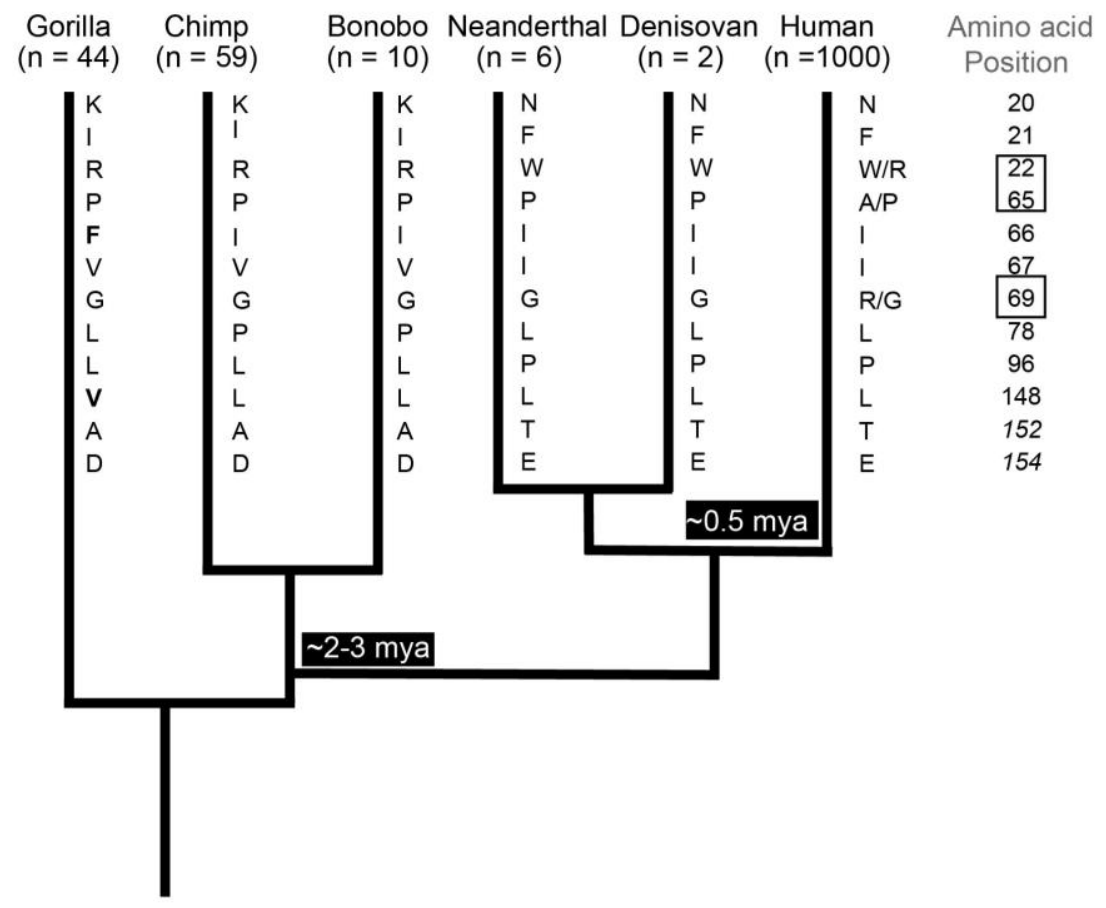

495 Figure 1: Human specific changes in CD33 are primarily present in the Sia binding V-set

496 domain. (A) Comparison of amino acid sequences of CD33 from humans and "great apes" was 497 performed using Conformational Analysis Tools software. The Great ape genomes included in the analysis are gorilla, chimpanzee and bonobo and were compared against the human protein as the 
499 template. The conservation of the sequence is indicated with yellow being the most and red being 500 the least conserved regions. Amino acids that are different from huCD33 are highlighted in pink. 501 Amino acids encoding the different CD33 domains are indicated above the sequence with different 502 colors corresponding to schematic in inset, namely, $\mathrm{V}$-set domain in green, $\mathrm{C} 2$ domain in light 503 blue, transmembrane domain in grey and cytoplasmic end in light pink. The flexible C-C' loop is 504 indicated in orange. Amino acids that are in contact with Neu5Ac in huCD33 are highlighted in 505 magenta and N-glycosylation sites in blue. (B) Phylogenetic analysis of the evolution of the 506 extracellular domains of CD33 proteins from human, Great apes and archaic genomes. The number 507 of genomes (n) for each group included in the analysis is indicated. Human and great ape CD33 508 sequences were compared with six Neanderthal and two Denisovan genomes. Amino acid changes 509 present in human CD33 were also present in the ancient genomes. The positions of the amino acids 510 that are different between human and the apes are mentioned, and the identity of the amino acid 511 present in the corresponding positions for each group is indicated by the single letter abbreviations 512 along the branch. Amino acids at positions 152 and 154 are within the C2 domain of CD33 protein 513 and italicized. Polymorphisms within the human population at positions 22, 65 and 69 of CD33 514 protein are indicated. Amino acids in gorilla CD33 at positions 66 and 148 are different from other 515 apes and are bold. Possible timeline for the diversion of the hominin lineage is indicated in the 516 tree. Length of the branches in the tree is not to scale. Mya = million years ago. 
A

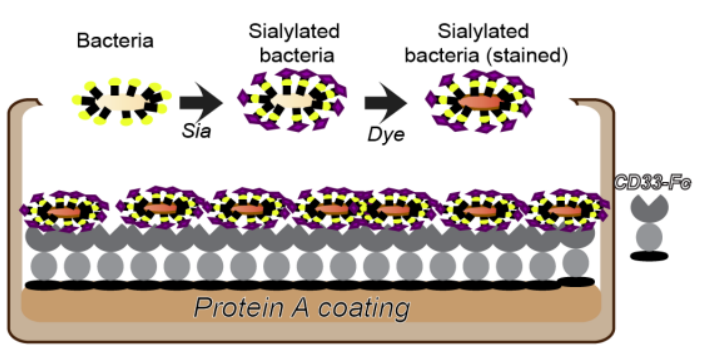

B

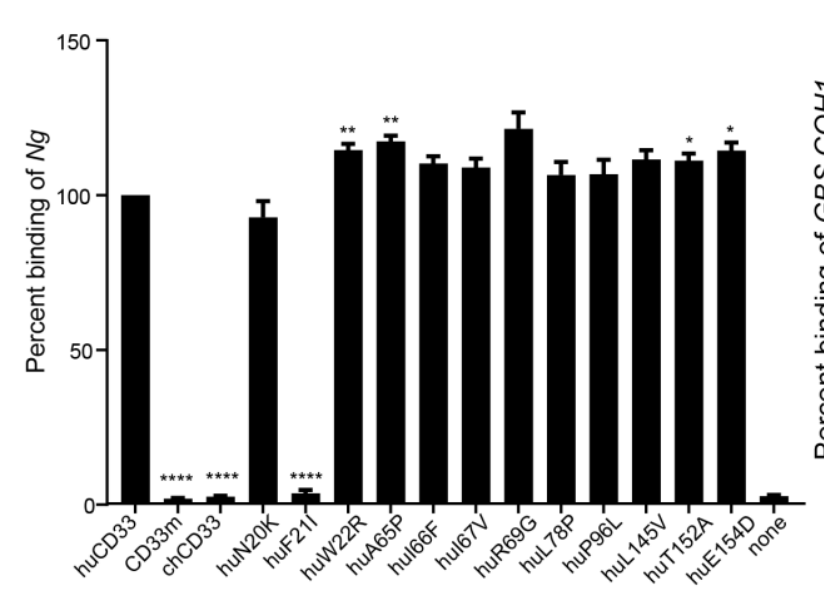

C

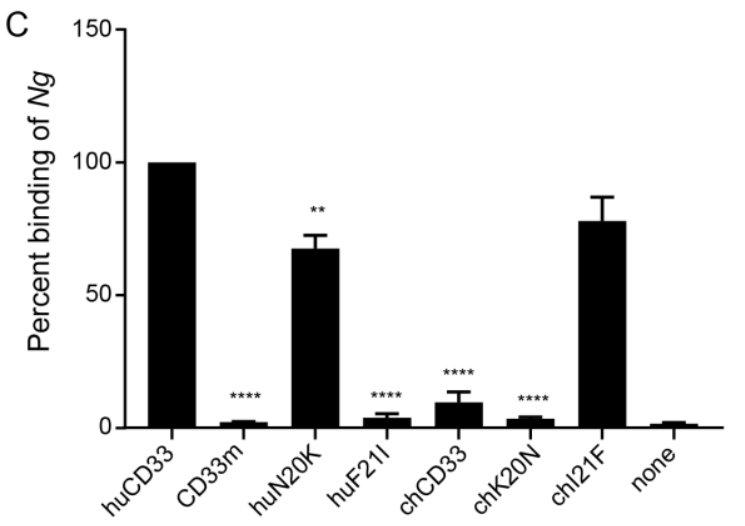

E

F
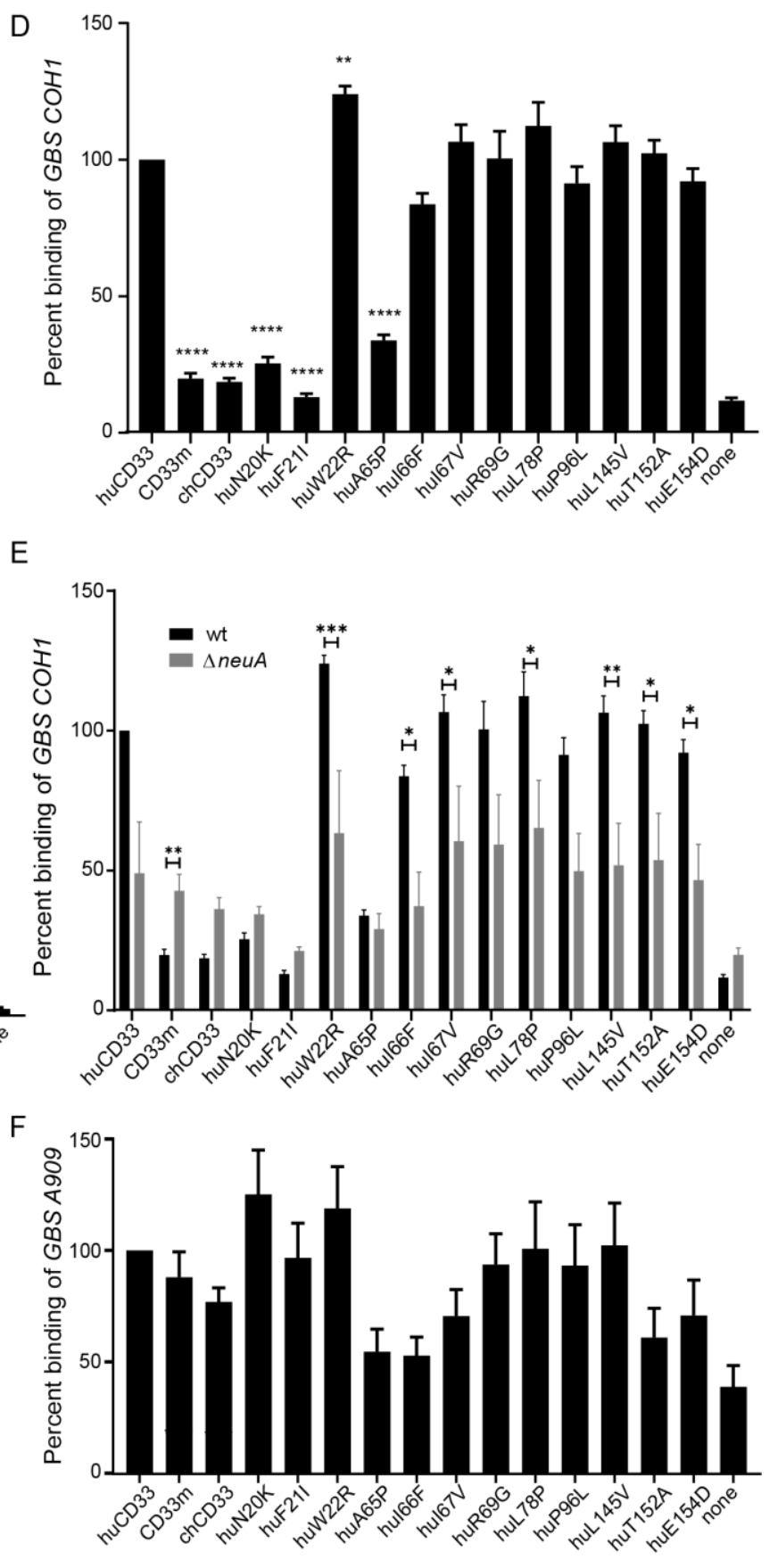

518 Figure 2: Human specific amino acid changes in CD33 affects bacterial binding. (A)

519 Schematic of the ELISA-based assay using recombinant CD33-Fc chimeric proteins immobilized

520 on protein A coated plates used to determine binding of the sialylated bacteria is shown. (B)

521 Binding of fluorescently labelled Neisseria gonorrhoeae $(\mathrm{Ng})$ was determined. The position of the

522 amino acid different from the wildtype human CD33 protein is indicated below each bar in the x-

523 axis. The bacterial binding to each individual CD33 mutant was normalized to the binding of 
524 wildtype human CD33 for that assay. "None" indicates no protein control for the background 525 bacterial binding to the plate. (B) Binding of Neisseria to immobilized recombinant CD33 proteins 526 containing the corresponding amino acid mutation (position 20 or 21) in either in human or chimp 527 CD33 protein backbone. (D) Binding of Group B Streptococcus (GBS) COH1 strain to different 528 CD33 mutant proteins in an ELISA based assay with immobilized recombinant CD33 proteins.

529 (E) Sialic acid dependence of the binding was determined using wildtype and $\triangle n e u A$ mutant strains 530 of COH1. (F) Interaction of CD33 proteins among different GBS strains was compared using A909 531 and COH1 strains. 'hu' indicates the corresponding amino acid change in human CD33 backbone 532 and 'ch' using chimp CD33. The graphs show the cumulative result from 3 independent 533 experiments, each done in triplicate. Statistical analysis was performed in Prism software using 534 one-way ANOVA with Durrett post comparison test. $*<0.01, * *<0.001, * * *<0.0001$. 


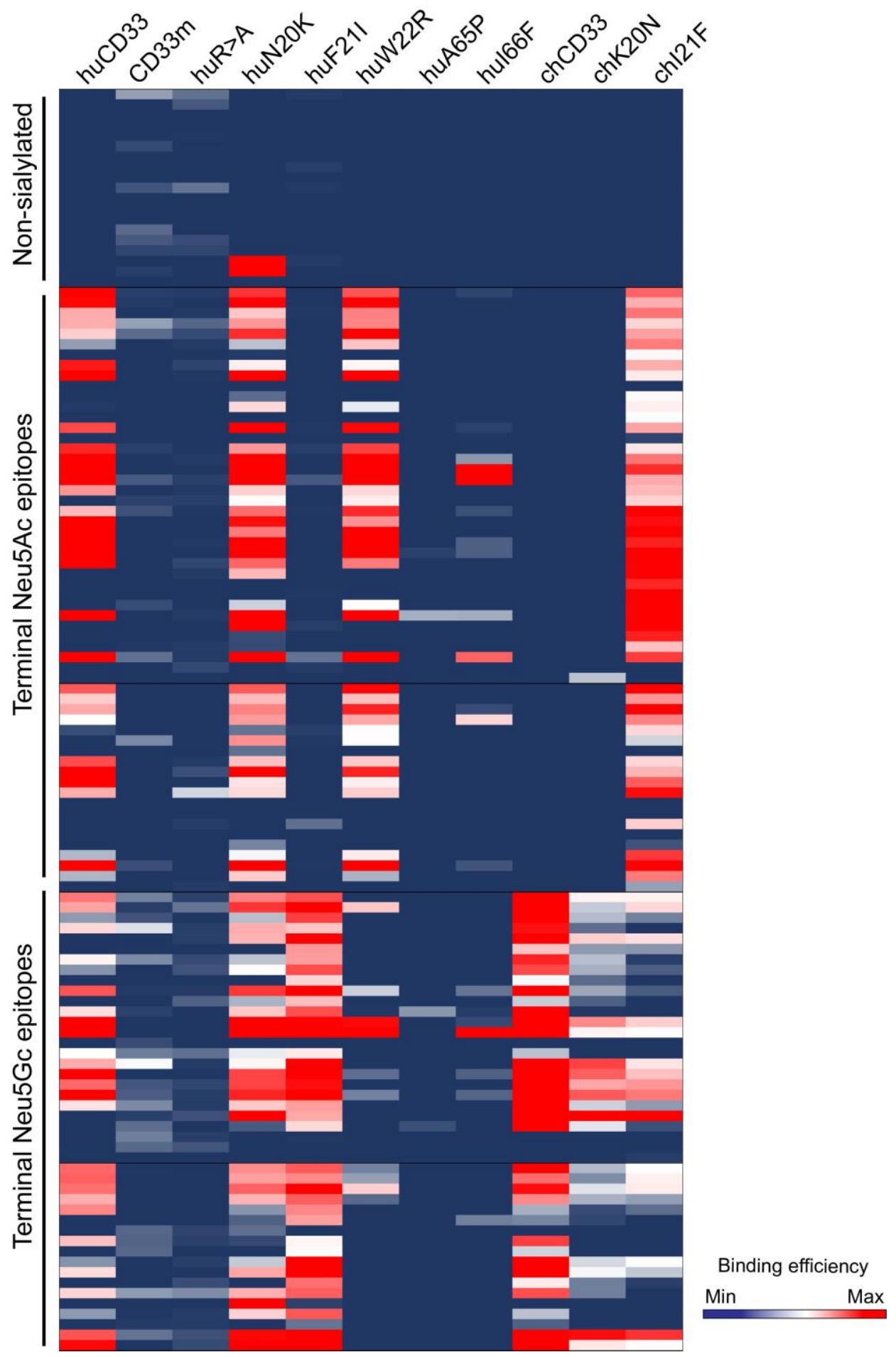

537 Figure 3: Single amino acid changes affect CD33 sialoglycan binding. Sialoglycan binding 538 profile of purified, soluble, recombinant CD33 proteins was determined using a sialoglycan 539 microarray containing defined, chemically synthesized glycans. Non-sialylated, Neu5Ac- and 
540 Neu5Gc-terminating glycans were grouped together in the heatmap as shown in the left. Each 541 column indicates the binding profile of the protein indicated on the top and each row represent a 542 distinct glycan. Blue indicates no binding and red indicates very strong binding preferences 543 characterized by an average relative fluorescence unit (RFU) of more than 90th percentile. The 544 result of the heatmap is summarized in Supplemental Figure S2 and the names of the individual 545 glycans are presented in Supplemental File S1. 

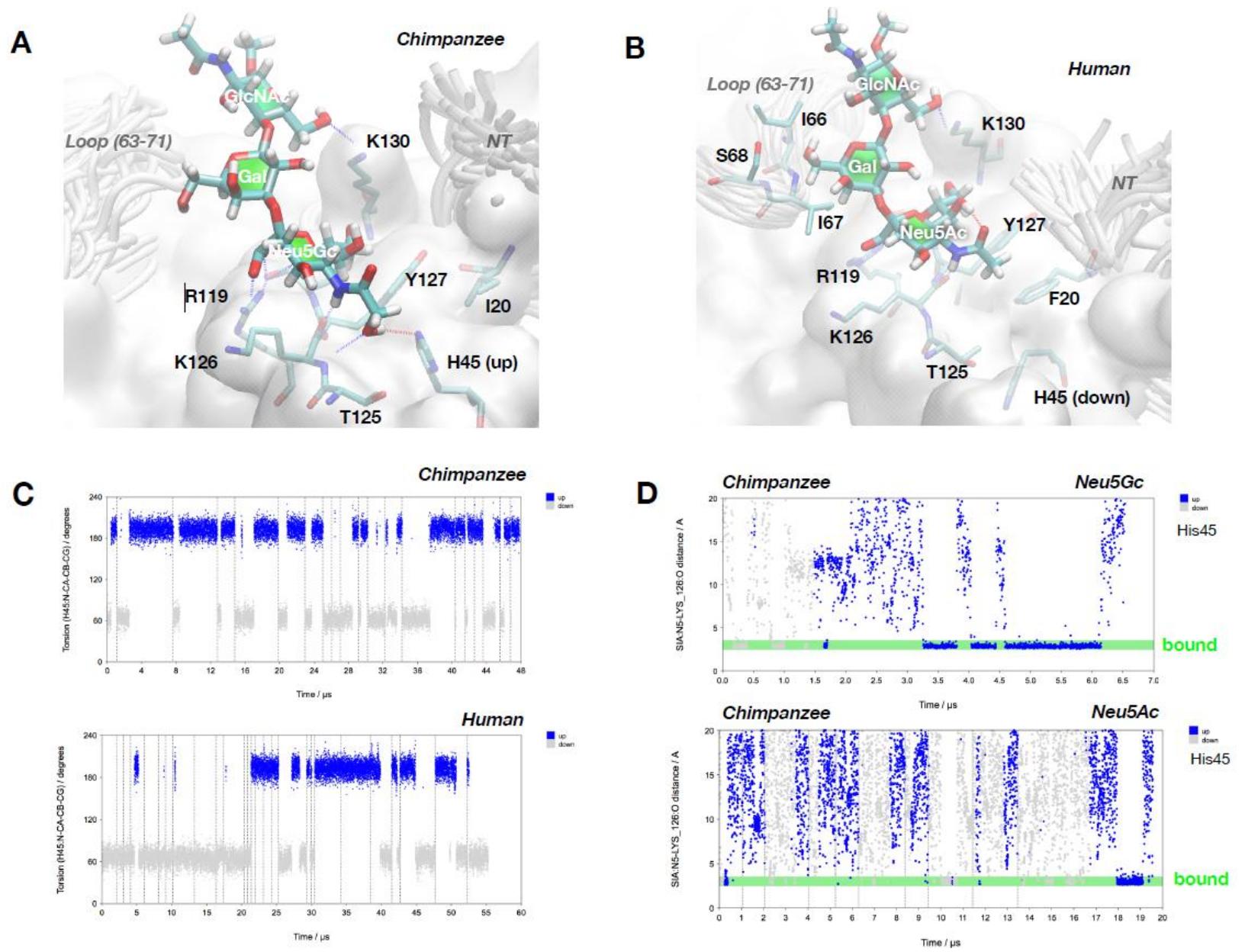

547 Figure 4: Structural modeling to understand the differential binding preference of human

548 and chimpanzee CD33 proteins. (A) 3D model of the complex between Neu5Gc $\alpha 2-3 \mathrm{Gal} \beta 1$ -

549 4GlcNAc $\beta O M e$ and chCD33. The increased affinity of Neu5Gc may be explained by

550 intermolecular hydrogen bonds involving the $\mathrm{OH}$-group of Gc. It should be noted that the number

551 of favorable interactions is maximal when His45 is in 'up' conformation. (B) 3D model of the

552 complex between Neu5Ac $\alpha 2-3 \mathrm{Gal} \beta 1-4 \mathrm{GlcNAc} \beta O M e$ and huCD33. The methyl group of Ac is

553 located in a small hydrophobic pocket formed by the side chains of Tyr127 and Phe20. It should

554 be noted that His45 is in 'down' conformation because otherwise - in the conformation shown -

555 the bulky side chain of Phe20 would overlap partly with His45 in 'up' conformation. (C) Molecular

556 dynamics of His45 side chain orientation. Accumulated MD trajectories of torsion angle N-C $\alpha$ -

$557 \mathrm{C} \beta-\mathrm{C} \gamma$ are shown. The 'up' conformation is present when torsion values are fluctuating around

558200 degrees and the 'down' conformation is characterized by values around 70 degrees. For

559 chimpanzee, it can be observed reproducibly that simulations started with His45 in 'down' 
560 conformation undergo a transition to the 'up' conformation on the microsecond timescale. In 561 contrast the 'down' conformation appears to be more stable in huCD33, which would make 562 Neu5Ac binding more likely. (D) MD simulation of unbiased binding and unbinding events of 563 Neu5Gc (top) and Neu5Ac (bottom) to chCD33. For Neu5Gc the lifetime of the complex is 564 significantly longer when His45 is in 'up' conformation, as can be seen from the $6.5 \mu \mathrm{s}$ MD 565 simulation shown on the top. Also, for Neu5Ac multiple binding and unbinding events occurred 566 on a timescale of about $20 \mu \mathrm{s}$, however in general (with one exception) the lifetimes of the 567 complexes formed are significantly shorter than for Neu5Gc. 

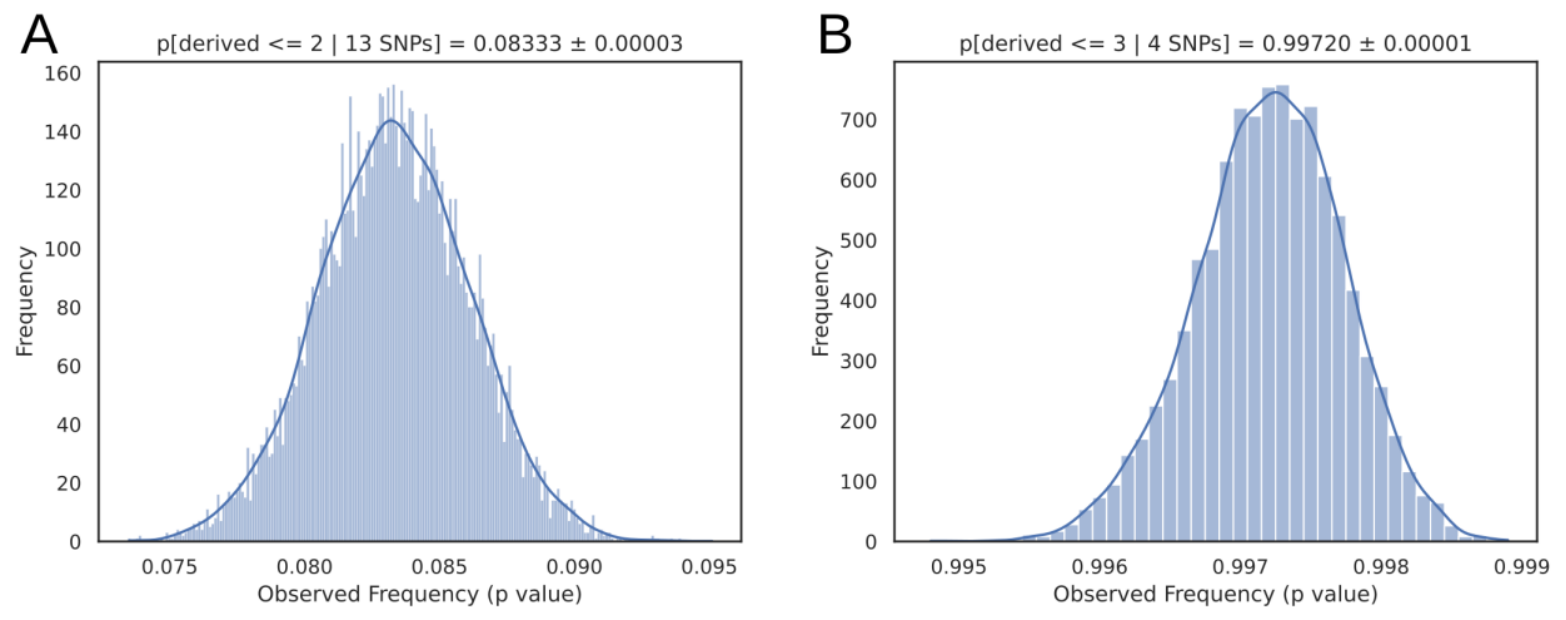

569 Figure 5: Resampling analysis with matched allele frequency SNPs from 1000 genomes

570 variants. Frequency distribution of SNPs with similar properties to the LOAD protective set (A)

571 and other Siglec SNPs (B). 


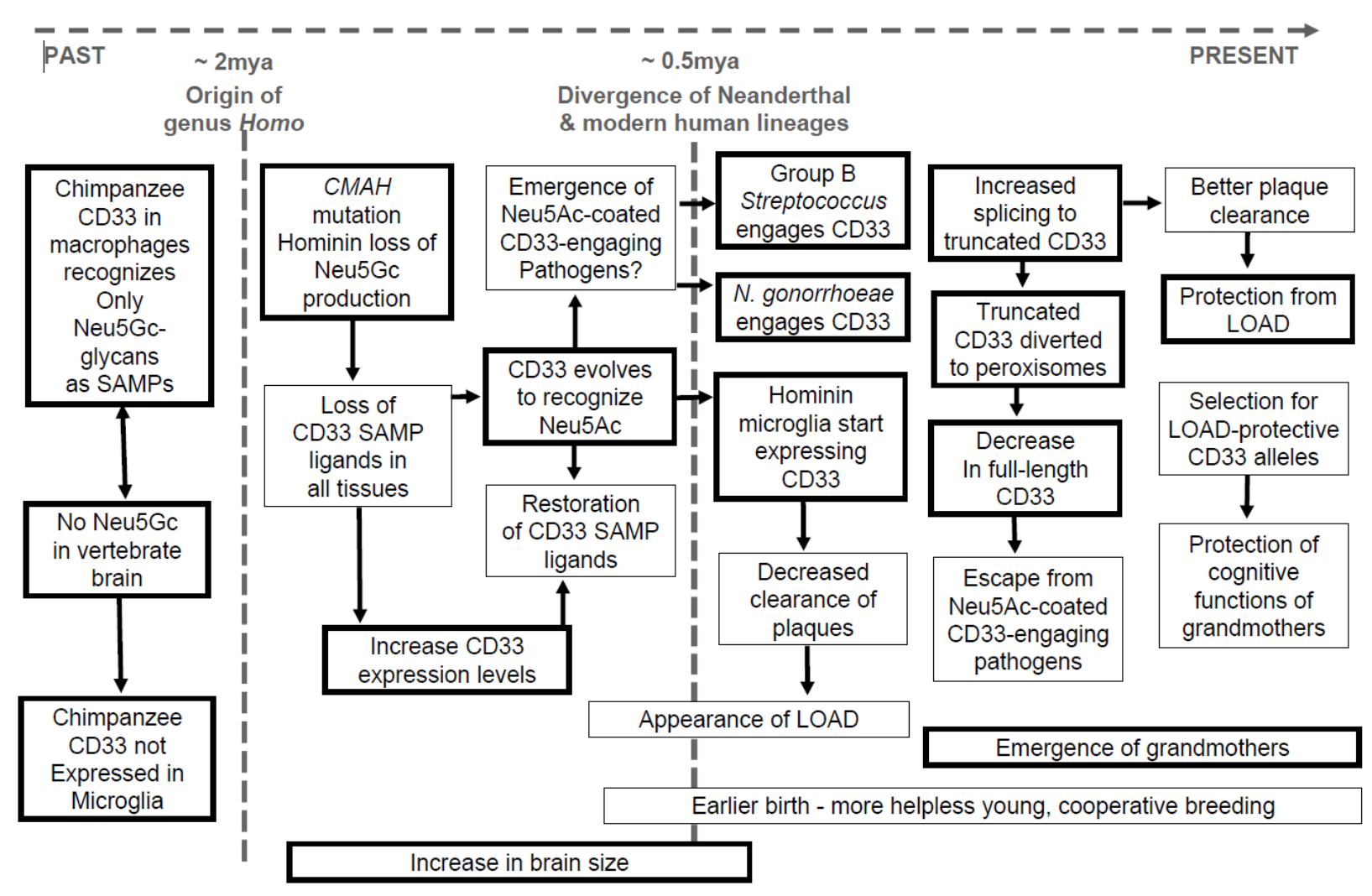

573 Figure 6: Scenario for evolution of human CD33 in relationship to cell surface sialic acids,

574 infectious disease, brain microglia and cognitive maintenance of grandmothers and other

575 elderly caregivers. This schematic presentation combines the known/likely facts (thick-outlined

576 boxes, including data from this manuscript) as well as suggested possibilities (thin-outlined boxes)

577 into the most likely evolutionary scenario for human-specific evolution of CD33. Starting from

578 the left, the likely chronological order of occurrence is indicated (by arrowheads) with the

579 approximate timeline on the top, along the dotted lines. '?' indicates our reasonable assumption

580 leading to the event. See text for further discussion. 
Table

583 Table 1: Gene variants directly or indirectly affecting cognitive function. Allele has the derived 584 allele as the lower, bolded entry. Archaic genotypes are reported for SNPs passing all quality 585 filters.

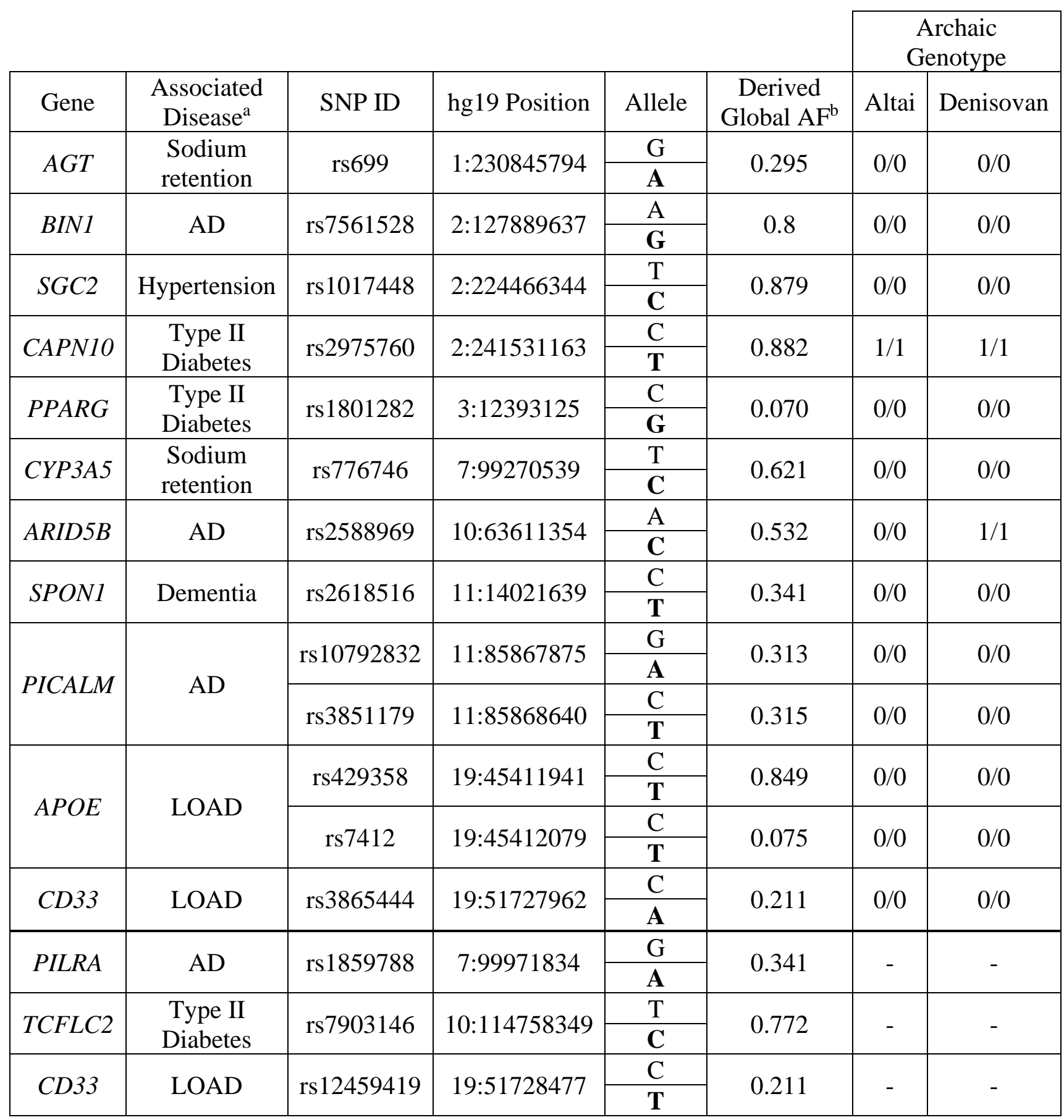

${ }^{a}$ See supplemental Table S1 for details and the primary literature citations.

587 bee supplemental Table S2 for the global population distribution. 
589 Bacterial culture and cell lines. The bacterial strains used were Neisseria gonorrhoeae F62 $\operatorname{lgtD}$ (generous gift from Sanjay Ram, University of Massachusetts Worcester), Group B Streptococcus

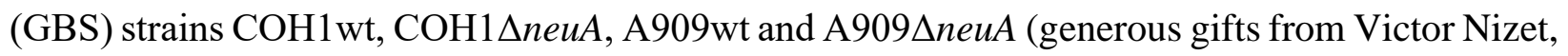

592 University of California San Diego). Neisseria were grown overnight on chocolate II agar plate and GBS on Todd Hewitt agar plate at $37^{\circ} \mathrm{C}$ and $5 \% \mathrm{CO}_{2}$ from the respective frozen glycerol stocks. Prior to the assay, GBS was grown in Todd Hewitt broth at $37{ }^{\circ} \mathrm{C}$ and $5 \% \mathrm{CO}_{2}$ without shaking. The E. coli $\mathrm{K} 1$ strain was grown in LB. For the CD33 protein purification, HEK293A cells were grown in DMEM media (Invitrogen) containing $10 \% \mathrm{FCS}$ at $37{ }^{\circ} \mathrm{C}$ and $5 \% \mathrm{CO}_{2}$.

Sialylation of Neisseria. Following overnight growth on chocolate agar plate, the bacteria were grown in $\mathrm{GC}$ broth supplemented with IsoVitaleX at $37^{\circ} \mathrm{C}, 5 \% \mathrm{CO}_{2}$ and shaking at $200 \mathrm{rpm}$ in presence or absence of $30 \mu \mathrm{M}$ CMP-Neu5Ac (Nacalai USA. Inc.) until OD600 equivalent to 0.4$600 \quad 0.5$.

601 Bacterial staining. Following appropriate growth, the bacteria were washed with pre-warmed HBSS and stained with $2 \mu \mathrm{M} \mathrm{SYTO13} \mathrm{(Thermo} \mathrm{Scientific)} \mathrm{for} 30 \mathrm{~min}$ at $37^{\circ} \mathrm{C}$ and shaking at 200 rpm in dark. After incubation, the stained bacteria were washed with HBSS and resuspended to a final concentration of OD600 $=1 / \mathrm{ml}$ in HBSS for the binding assay.

605 Generation of CD33 mutant proteins. A genomic fragment (1228 bp) of human or Chimpanzee CD33(M), including the first 4 exons (2 Ig domains) was fused with pcDNA3.1(-) containing a Cterminal FLAG (EK) sequence followed by a hIgG1-Fc genomic fragment (hinge + 2 Ig-like domains) and described elsewhere [67, 68]. Sixteen mutant variants were made from either construct above using New England Biolabs Q5 site directed mutagenesis Kit according to the manufacturer's instructions (Supplemental Table 3). Mutagenesis primers listed were designed using NEBaseChanger software.

612 Truncated CD33(CD33m)_EK_Fc Construction: U937 cells were cultured in RPMI 1640

613 supplemented with 10\% FCS. Total mRNA was isolated using Qiagens Oligotex Direct mRNA 614 Mini Kit according to the manufacturer's instructions. CD33m was amplified by PCR using 615 SuperScript III One-Step RT-PCR (Invitrogen) and Gene-specific primers 5'616 TTATATGCTAGCGCCACCATGCCGCTGCTGCTACTGCTGC-3', NheI site underlined and 617 5'-GCGCGCGATATCATGAACCACTCCTGCTCTGGTCTCTTG-3', EcoRV site underlined. 618 PCR products were run on $2 \%$ agarose gel and the $396 \mathrm{bp}$ bands corresponding to CD33(m) were 
619 excised and cut with NheI/EcoRV restriction enzymes. Digested bands were sub-cloned into 620 pcDNA3.1(-) containing a C-terminal FLAG (EK) sequence followed by a hIgG1-Fc genomic 621 fragment (hinge +2 Ig-like domains).

622 Purification of CD33 mutants. Transfection supernatants were collected and spun down at 500 $623 \mathrm{~g}$ for 5 mins to remove cellular debris. The $\mathrm{pH}$ of each supernatant was adjusted to $\mathrm{pH} 8.0$ for 624 optimal binding of protein A-Sepharose beads to hIgG Fc fusion protein. Protein A-Sepharose 4 625 Fast Flow suspension (GE Healthcare) was washed with Tris-Buffered Saline (TBS) pH 8.0, and 626 a 1:500 ratio of beads:media added to each supernatant. Each tube was subsequently incubated for $62724 \mathrm{hrs}$ on a roller in the cold-room. After 24 hours supernatants plus beads were transferred to 628 disposable columns until all liquid has run thru. Beads were washed 3x with TBS pH 8.0 before 629 being eluted directly in $0.3 \mathrm{ml}$ of $1 \mathrm{M}$ Tris- $\mathrm{HCl} \mathrm{pH} 8.0$ using $0.1 \mathrm{M}$ Glycine Buffer $\mathrm{pH} 2.8$. Each 630 eluate was put into an Amicon Ultra-15 filter unit with MWCO $30 \mathrm{~K}$ for each full length CD33631 EK_Fc variant and MWCO $10 \mathrm{~K}$ for huCD33m-EK_Fc. Tubes were centrifuged at 4,000 g for 20 632 mins. Run-through was discarded and the columns washed 3x with TBS pH 8.0. After the last 633 wash, each retentate was recovered from the column and stored at $-80^{\circ} \mathrm{C}$.

634 Binding assay with the bacteria. Bacterial binding with the CD33 proteins were done with the 635 recombinant Fc-chimeric proteins of CD33. Briefly, protein A coated black 96-well plate (Pierce, 636 Thermo Scientific) was washed thrice with TBS containing 0.05\% Tween 20 (TBS-T) and coated 637 with $200 \mathrm{ng} / \mathrm{well}$ of the respective CD33 protein diluted in $200 \mathrm{mM}$ Tris- $\mathrm{HCl} \mathrm{pH} 8.0,150 \mathrm{mM}$ $638 \mathrm{NaCl}$ and $1 \% \mathrm{BSA}$ at $4{ }^{\circ} \mathrm{C}$ overnight. Following incubation, the coated plate was washed with 200 $639 \mathrm{mM}$ Tris $\mathrm{pH} 8.0,150 \mathrm{mM} \mathrm{NaCl}$ to eliminate the unbound proteins. Stained bacteria equivalent to 640 OD600 $=0.1$ was added to each well of the plate and allowed to interact with the proteins for 30 $641 \mathrm{~min}$ at $37^{\circ} \mathrm{C}$ and $5 \% \mathrm{CO}_{2}$ without shaking. Following incubation, the plate was washed with TBS642 T to eliminate any unbound bacteria and the residual fluorescence was measured upon excitation 643 at $488 \mathrm{~nm}$ and emission at $530 \mathrm{~nm}$. The data were analyzed using the excel and Prism software.

644 Evolutionary analysis and Detection of positive selection. The protein coding sequences of 645 CD33 were aligned using CLUSTAL W program implemented in MEGA7 and then back 646 translated to obtain a codon alignment. The phylogenetic tree of CD33 protein coding sequences 647 were reconstructed with neighbor-joining method which was implemented in MEGA7 (Figure 1), 6481000 bootstrap replicates [69]. The unrooted neighbor joining tree was used for the subsequent 649 analysis. 
650 VCF files were accessed from ftp://ftp.1000genomes.ebi.ac.uk/vol1/ftp/release/20130502/ for 6511000 genomes project, http://cdna.eva.mpg.de/neandertal/altai/AltaiNeandertal/VCF/ for Altai

652 Neanderthal and http://cdna.eva.mpg.de/denisova/VCF/hg19_1000g/ for Denisovan. Quality 653 filters were obtained from https://bioinf.eva.mpg.de/altai_minimal_filters/ for Altai and 654 Denisovan. Individuals in 1000 genomes datasets were assigned to populations using 655 http://ftp.1000genomes.ebi.ac.uk/vol1/ftp/technical/working/20130606_sample_info/20130606_s

656 ample info.txt. First, all vcf files were filtered by intersecting with the quality bed files using 657 bedtools intersect (v2.28.0). The filtered vcf files were then combined, per chromosome, to match 658 their position, reference and alternative allele using a custom python script. VCF information was 659 retained along with per-population allele frequencies and archaic genotypes. Next, ancestral alleles 660 were obtained from ensembl (https://rest.ensembl.org/variation/homo_sapiens) by querying each 661 SNP id and appending to the joint vef entries. The joint vcf files were used as input for further 662 processing in a jupyter notebook to perform resampling analysis. Each SNP of interest is used to 663 select collections of SNPs with matching global allele frequencies, +/- 0.004, or +/- 2 observed 664 haplotypes. A single draw consists of selecting one SNP from each collection to produce a 665 simulated observation and the number of SNPS with derived archaic haplotypes are recorded. 666 After 10,000 such draws, the faction of draws with fewer or equal numbers of derived SNPs is 667 used to produce a p-value estimate. The process is repeated 10,000 times to produce a histogram 668 and provide a confidence estimate on the reported p values (+/- SEM). Methods to replicate the 669 analysis can be found on Code Ocean.

670 Non-synonymous/ synonymous substitution ratios $(\omega=\mathrm{dN} / \mathrm{dS}$, or $\mathrm{Ka} / \mathrm{Ks})$ have become a useful 671 means for quantifying the impact of natural selection on molecular evolution. In general, the ratio $672 \omega=\mathrm{dN} / \mathrm{dS}$ is less than one if the gene is undergoing purifying selection, equal to one if the gene 673 is evolving neutrally, and greater than one if positive selection has accelerated the fixation of non674 synonymous substitutions that resulted in amino acid changes. The pair-wise computation of $675 \mathrm{Ka} / \mathrm{Ks}$ between $\mathrm{V}$-set exon of each species were performed using the program DnaSp v.0 6.0. The 676 initial unrooted tree fed to the program in the format of Newick was: ((Chimpanzee5:0.00000000, 677 Bonobo:0.00000000):0.00222522, Gorilla:0.00979959, Human:0.01351877).

678 Molecular Simulation. Starting structures of the V-type domain (residues 18-142) of CD33 were 679 built based on PDB entries 5j0b (A chain) and 6d49 using the graphical interface of YASARA 680 [70]. The two structures differ significantly with respect to the conformation of the C-C' loop 
681 (residues 63-71, compare Supplemental Figure S3). A single mutation (G69R) was introduced into $6825 \mathrm{j} 0 \mathrm{~b}$ to build CD33(human). The initial 3D models of chCD33 were built by swapping residues: 683 N20K, F21I, W22R, A65P, I67V, R69G (in 6d49), L78P, P96L. An N-glycan core (M3) was 684 attached to Asn100. The side chain of His45 was modeled in two conformations (compare Figure 685 6): 'down' (as in PDB entry 6d49) and 'up' (as present in PDB entries 5ihb or 5j06 chains A). The systems were solvated in $0.9 \% \mathrm{NaCl}$ solution $(0.15 \mathrm{M})$ and simulations were performed at $310 \mathrm{~K}$ using periodic boundary conditions. The box size was rescaled dynamically to maintain a water 688 density of $0.996 \mathrm{~g} / \mathrm{ml}$. Additionally systems were built that contain five molecules of Neu5GcaOMe or Neu5AcaOMe distributed in the simulation box which allowed to simulate binding events. Simulations were performed using YASARA with GPU acceleration [71]. In total $27 \mathrm{MD}$ trajectories were sampled for huCD33 and 20 for chCD33, most of them covering a microsecond timescale (compare Supplemental Figure S4). Conformational Analysis Tools (CAT, http://www.md-simulations.de/CAT/) was used for analysis of trajectory data, general data processing and generation of scientific plots. VMD [72] was used to generate molecular graphics. Sialoglycan microarray. The sialoglycan microarray experimental method was adopted from the literature reported earlier [73, 74]. Chemoenzymatically synthesized sialoglycans were quantitated utilizing DMB-HPLC method [75] and $10 \mathrm{mM}$ aqueous stock solutions were prepared. Next, the glycans were diluted to $100 \mu \mathrm{M}$ in $300 \mathrm{mM}$ Na-phosphate buffer (pH 8.4) and printed in quadruplets on NHS-functionalized glass slides (PolyAn 3D-NHS; catalog\# PO-10400401) using an ArrayIt SpotBot® Extreme instrument. The slides were blocked using 0.05M ethanolamine solution in 0.1 M Tris-HCl ( $\mathrm{pH} 9.0$ ), washed with warm Milli-Q water and dried. Printed slides were fitted in a multi-well microarray hybridization cassette (ArrayIt, CA) and rehydrated using $400 \mu \mathrm{l}$ of ovalbumin $(1 \% \mathrm{w} / \mathrm{v}, \mathrm{PBS})$ for one hour in a humid chamber with gentle shaking. The solution was discarded followed by the addition of $400 \mu$ l solution of the CD33 protein $(30 \mu \mathrm{g} / \mathrm{ml}$ in PBS with $1 \% \mathrm{w} / \mathrm{v}$ ovalbumin) in the individual well. The slides were incubated for $2 \mathrm{~h}$ at ambient temperature with gentle shaking followed by washing with PBS-Tween $(0.1 \% \mathrm{v} / \mathrm{v})$ and PBS. The wells were then treated with Cy3-conjugated goat anti-human $\operatorname{IgG}$ (1:500 dilution in PBS), incubated for $1 \mathrm{~h}$ in a dark humid chamber with gentle shaking. After washing and drying, the slides were scanned using a Genepix 4000B scanner (Molecular Devices Corp., Union City, CA) at wavelength $532 \mathrm{~nm}$. Data analysis was performed using the Genepix Pro 7.3 software (Molecular Devices Corp., Union City, CA). 


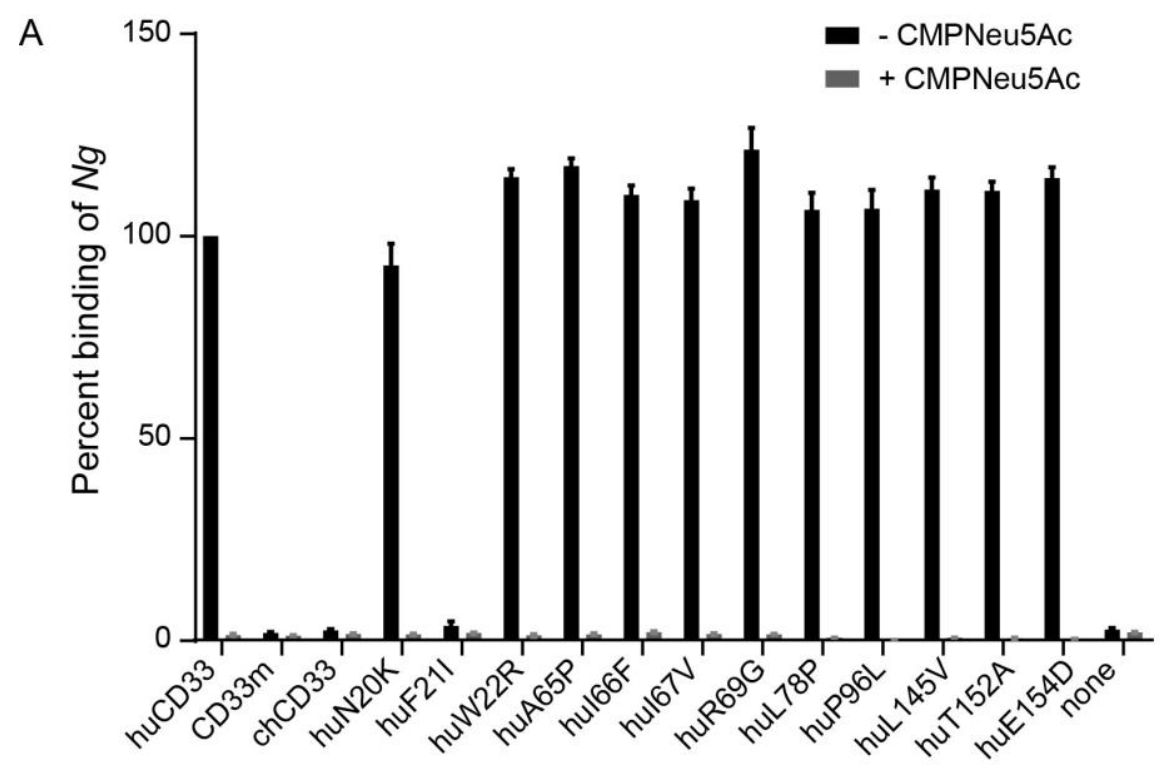

B

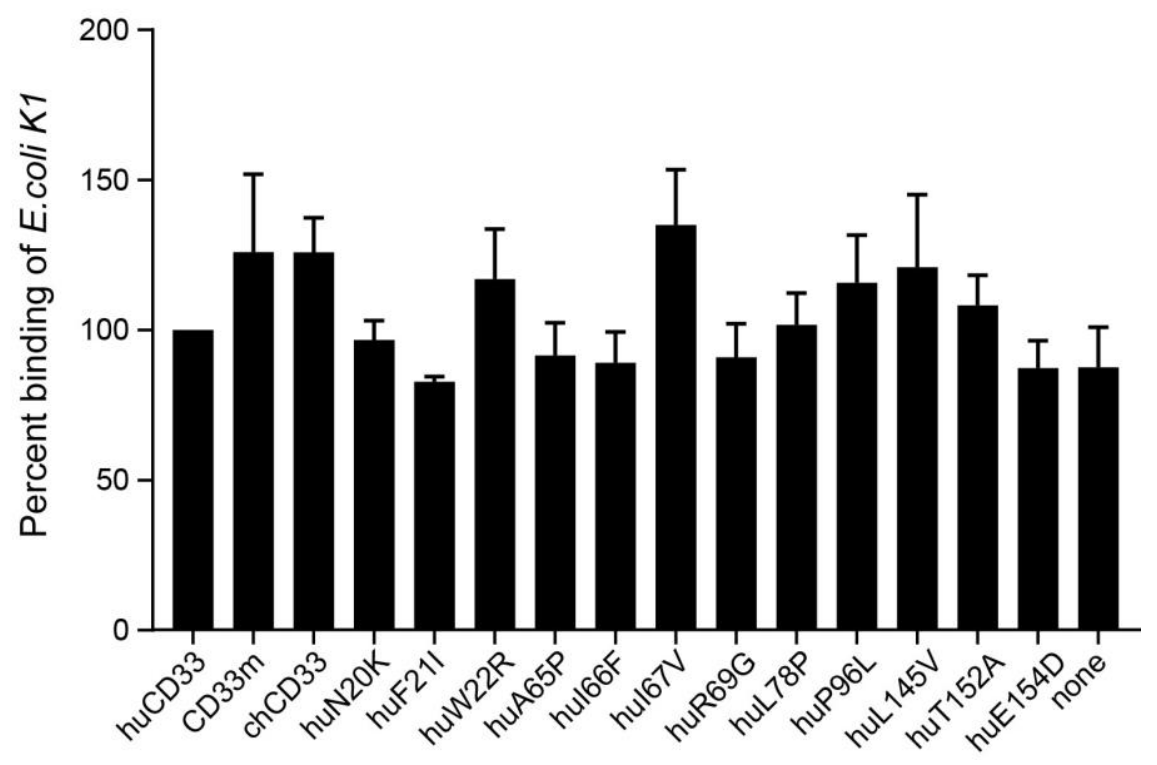

717 Supplemental Figure S1: While E. coli does not bind CD33, human CD33 binding by

718 Neisseria is Sia-dependent. (A) Binding of $\mathrm{Ng}$ with wildtype or mutant CD33 proteins was

719 determined in the same manner as in Figure 2A. The bacteria for the assay were either grown in

720 presence (+) or absence (-) of exogenous CMP-Neu5Ac as indicated in the legend. All the binding

721 was normalized to wildtype human CD33 binding. Cumulative data from 2 independent

722 experiments, each done in triplet is presented. (B) Binding of E. coli $\mathrm{K} 1$ was determined using the 
bioRxiv preprint doi: https://doi.org/10.1101/2021.11.26.470088; this version posted November 26, 2021. The copyright holder for this preprint (which was not certified by peer review) is the author/funder. All rights reserved. No reuse allowed without permission.

723 different CD33 proteins. None of the proteins showed any increased binding to the bacteria relative

724 to no protein (control) containing blank well, indicating that there is no binding of the bacteria 725 with the protein. 


\begin{tabular}{|c|c|c|c|c|c|c|c|c|c|c|c|c|}
\hline Sialic acid & Linkage & 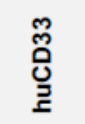 & 总 & $\begin{array}{l}\mathbb{\Lambda} \\
\hat{\alpha} \\
\text { है }\end{array}$ & $\begin{array}{l}\text { ્ㅗ․ } \\
\text { ્ָ. } \\
\text { E }\end{array}$ & $\underset{\text { ড̦ }}{\overline{\mathbf{E}}}$ & $\begin{array}{l}\underset{N}{\mathbb{N}} \\
\underset{\Xi}{\Xi}\end{array}$ & 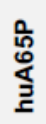 & $\begin{array}{l}\stackrel{4}{0} \\
\frac{0}{E}\end{array}$ & 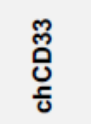 & 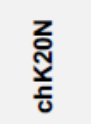 & $\frac{\frac{u}{\frac{\pi}{5}}}{\frac{c}{0}}$ \\
\hline Non-Sia & & - & - & - & $-*$ & - & - & - & - & - & - & - \\
\hline \multirow{3}{*}{ Neu5Ac } & $\alpha 2-3$ & ++ & - & - & ++ & - & +++ & - & $-*$ & - & - & ++ \\
\hline & $\alpha 2-6$ & +++ & - & - & +++ & - & +++ & - & - & - & - & +++ \\
\hline & $\alpha 2-8$ & $-{ }^{\circ}$ & - & - & + & - & -0 & - & - & - & - & +++ \\
\hline $\mathrm{Neu} 4,5 \mathrm{Ac}_{2}$ & $\alpha 2-3$ & + & - & - & + & - & + & - & - & - & - & +++ \\
\hline \multirow{2}{*}{ Neu5,9Ac 2} & $\alpha 2-3$ & ++ & - & - & $-1+$ & - & + & - & - & - & - & + \\
\hline & $\alpha 2-6$ & $-1+$ & - & - & + & - & $-1+$ & - & - & - & - & +++ \\
\hline Neu5Ac8Me & & - & - & - & - & - & - & - & - & - & - & - \\
\hline \multirow{3}{*}{ Neu5Gc } & $\alpha 2-3$ & + & - & - & ++ & +++ & $-\circ$ & - & -* & +++ & $-/+$ & - \\
\hline & $\alpha 2-6$ & +++ & - & - & +++ & +++ & - & - & - & +++ & +++ & ++ \\
\hline & $\alpha 2-8$ & - & - & - & - & ++ & - & - & - & +++ & ++ & ++ \\
\hline Neu4Ac5Gc & $\alpha 2-3$ & ++ & - & - & ++ & +++ & - & - & - & ++ & - & $-/+$ \\
\hline \multirow{2}{*}{ Neu5Gc9Ac } & $\alpha 2-3$ & + & - & - & + & +++ & - & - & - & +++ & - & - \\
\hline & $\alpha 2-6$ & ++ & - & - & +++ & +++ & - & - & - & + & + & + \\
\hline $\mathrm{Neu} 5 \mathrm{G} c^{\mathrm{Me}}$ & & - & - & - & - & - & - & - & - & - & - & - \\
\hline $\begin{array}{l}\text { Ganglioside } \\
\text { type }\end{array}$ & & - & - & - & - & - & - & - & - & - & - & $-\circ$ \\
\hline
\end{tabular}

727 Supplemental Figure S2: Summarized result of CD33 sialoglycan binding. The results of the

728 sialoglycan microarray binding of different wildtype and mutant CD33 proteins presented in

729 Figure 3 are summarized here. A differential sialoglycan binding preference was observed when

730 wildtype and mutant human/chimpanzee CD33 proteins were tested on the microarray. Binding is 731 annotated with a positive (+) symbol and the strength of the binding is indicated by the number of 732 the symbols. +++ indicates a very strong binding. Negative (-) symbol implies non-binding and 733 /+ indicates very faint interaction. In some cases, only a few sulfated glycans showed strong 734 binding signal (indicated with asterisk). Degree $\left({ }^{\circ}\right)$ symbols indicate binding with a very few 735 numbers of glycans only. Linkage indicates the nature of the glycosidic bond of the terminal Sia 736 to the underlying glycan. 


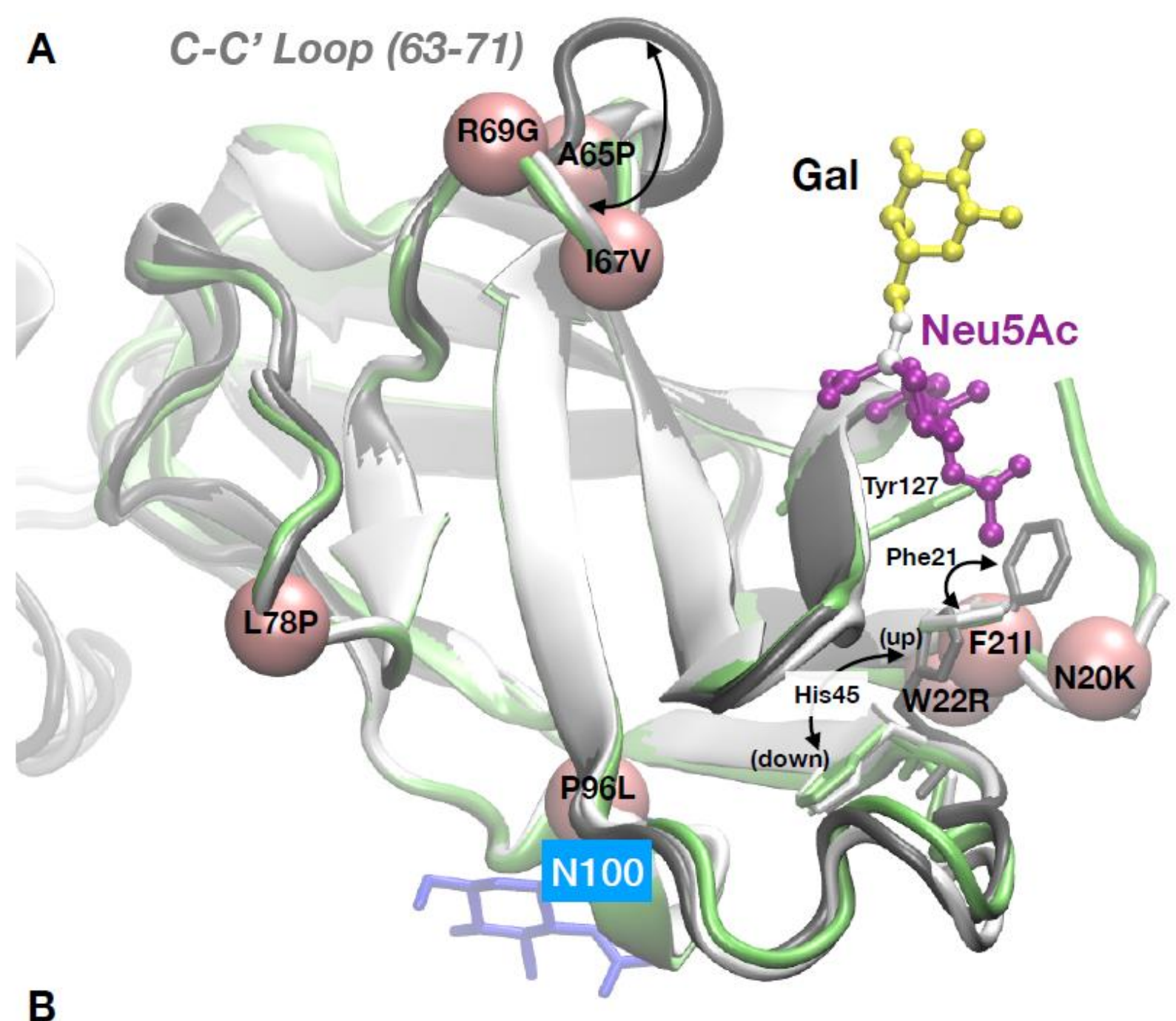

$\mathrm{B}$

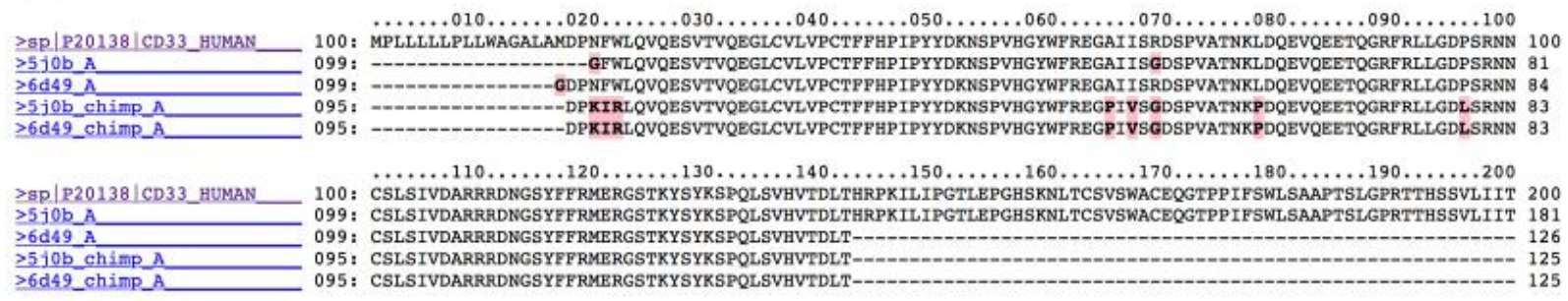

738 Supplemental Figure S3: Structure and dynamics of CD33. (A) Examples of x-ray structures 739 of huCD33. PDB entries 5ihb (chain A: dark grey, chain B: white), $6 \mathrm{~d} 49$ (lime). The dynamics of

740 the C-C' loop and residues Phe21 and His45 are indicated. Positions of mutations present in

741 chimpanzee are labeled on the pink spheres. (B) Amino acid sequences. 1: CD33 human (Uniprot),

742 2-3: PDB entries used for modeling. 3-4: sequences of the chCD33 models. 

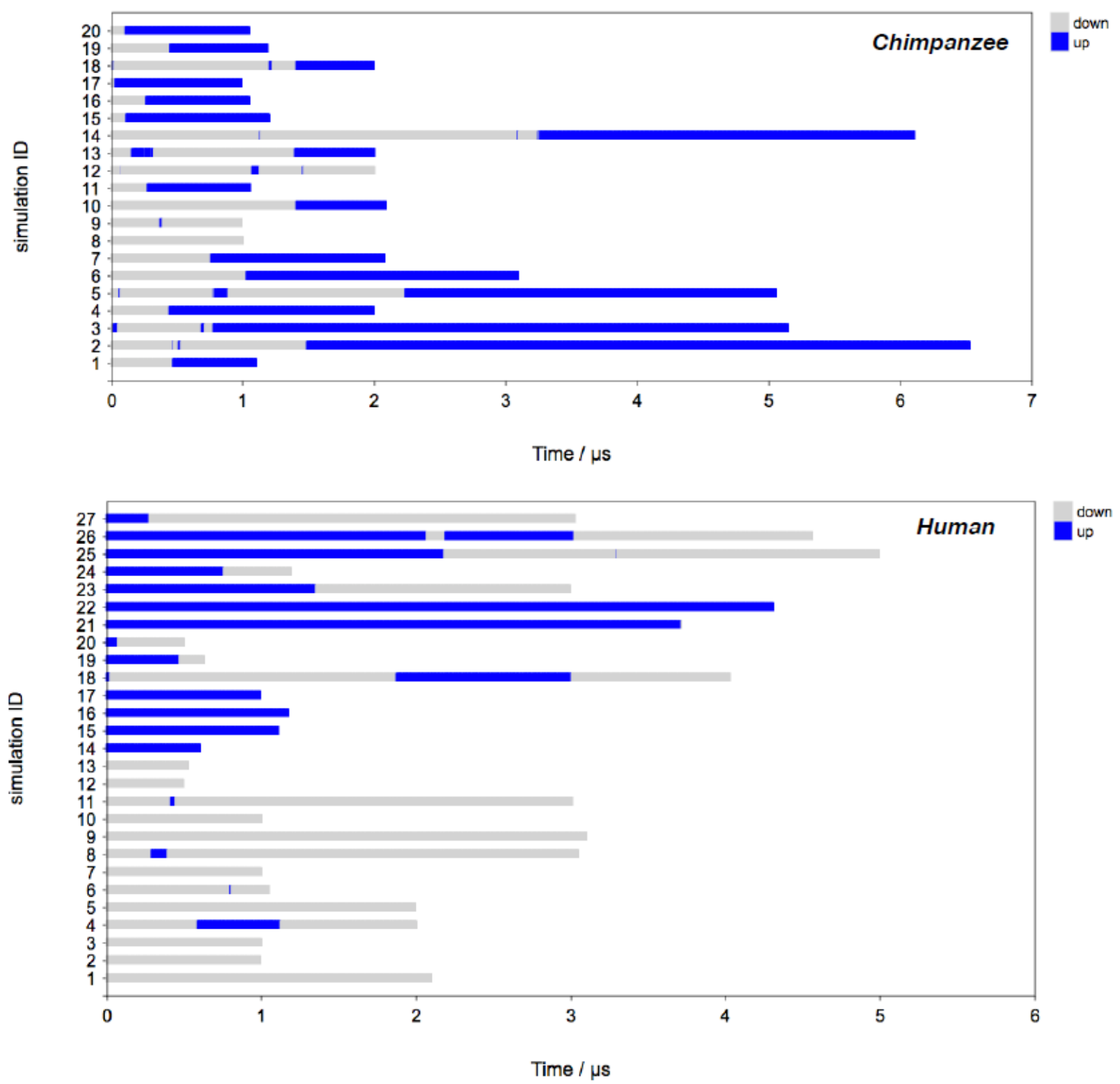

744 Supplemental Figure S4: MD trajectories of up/down states of Histidine at position 45 (His

745 45). Molecular dynamics of His45 side chain orientation. Individual MD trajectories of 746 'up'(blue)/‘down'(grey) conformational states are shown. For chCD33, it can be observed

747 reproducibly that simulations started with His45 in a 'down' conformational state undergo a 748 transition to the 'up' conformational state on the microsecond timescale. Therefore, it may be 749 concluded that chCD33 exists mainly with His45 in an 'up' orientation, which would be favorable 750 for binding of Neu5Gc. For huCD33, both conformational states can exist for multiple $\mu$ s, which 
bioRxiv preprint doi: https://doi.org/10.1101/2021.11.26.470088; this version posted November 26, 2021. The copyright holder for this preprint (which was not certified by peer review) is the author/funder. All rights reserved. No reuse allowed without permission.

751 explains why huCD33 can bind to Neu5Ac (preferably binds when His45 is 'down') and Neu5Gc 752 (preferably binds when His45 is 'up'). 

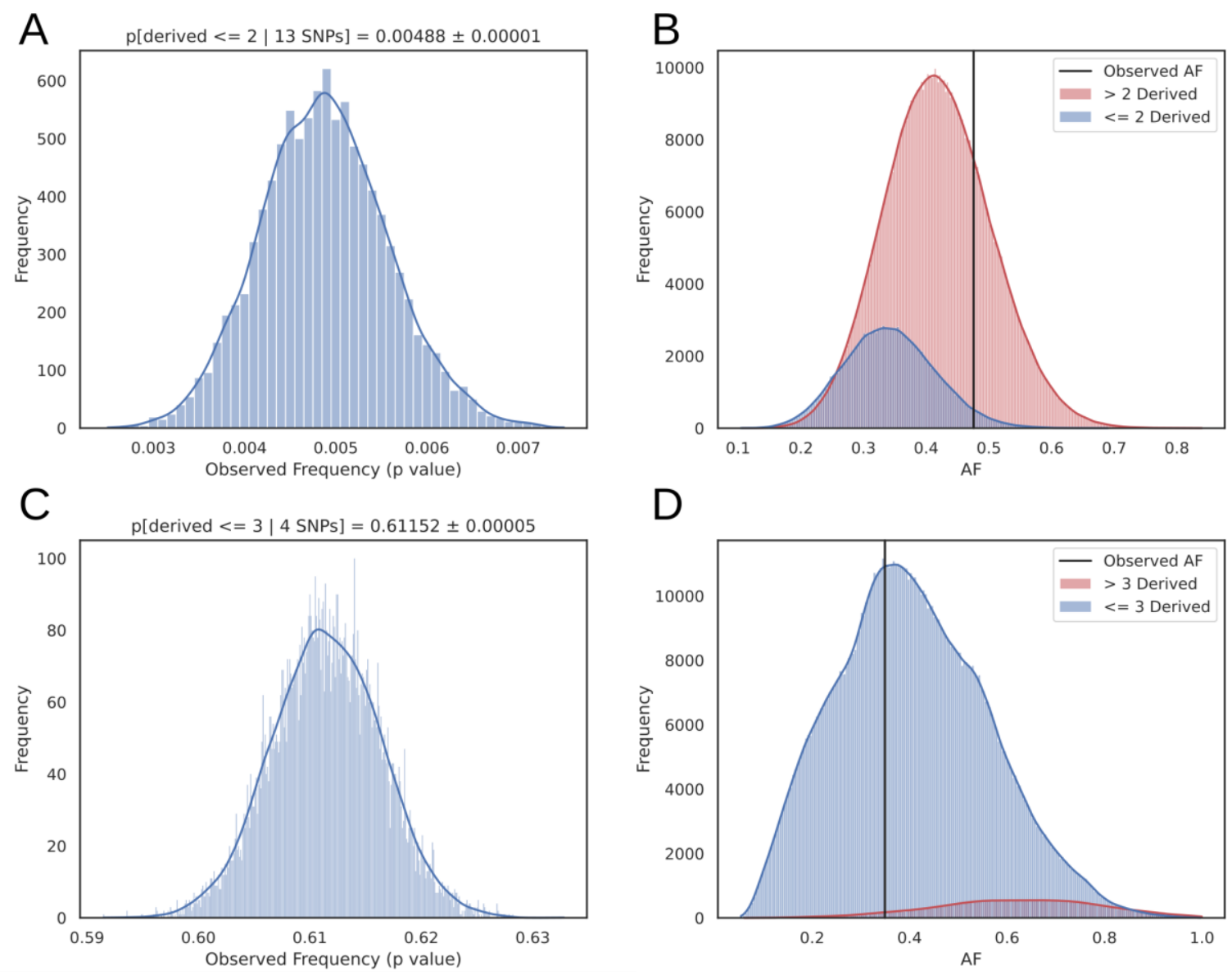

Supplemental Figure S5: Resampling analysis of the 5.9 million SNPs from 1000 genomes variants. As an alternative to matching AF directly, the set of filtered SNPs were further restricted to those with a derived population frequency greater than 0.05 resulting in a universe of 5.9 million

758 SNPs. We estimated the probability of observing at most two SNPs derived in either the 759 Neanderthal or Denisovan reference genomes and a mean allele frequency as large as the empirical 760 variants of interest ( $\mathrm{AF}=0.476)$. By randomly drawing SNPs, we found that the probability of 761 observing 13 SNPs with such as high global allele frequency and lack of derived alleles in archaic 762 genomes to be highly unusual ( $p$-value $=0.00487 \pm 0.00001)(\mathbf{A})$. The low frequency is driven by 763 two factors, as shown in (B). Most of the SNPs sampled have more than two archaic-derived SNPs 764 (red curve). Of those with fewer than two archaic-derived SNPs, the overall allele frequency is 765 typically low compared to the target set. With other Siglec SNPs, resampling captures similar 766 properties (C and $\mathbf{D})$, indicating the LOAD protective set does not represent a random sampling 767 from the genome. 


\begin{tabular}{|c|c|c|c|c|}
\hline Gene & $\begin{array}{l}\text { Associated } \\
\text { disease }\end{array}$ & SNP ID & Description & References \\
\hline$C D 33$ & LOAD & $\begin{array}{c}\mathrm{rs} 12459419, \\
\mathrm{rs} 3865444\end{array}$ & This study for details & {$[16,47]$} \\
\hline$A P O E$ & LOAD, CVD & $\begin{array}{l}\text { rs7412, } \\
\text { rs429358 }\end{array}$ & $\begin{array}{l}\text { Encodes plasma protein APOE, is polymorphic in humans. } \\
\text { Three alleles (E2, E3, E4) encode proteins with distinct } \\
\text { affinity for lipoprotein particles. The ancestral E4 allele is } \\
\text { associated with highest LOAD risk, and increased } \\
\text { atherosclerosis and vascular dementia. The derived alleles } \\
\text { E2 and E3 seems protective against LOAD, with the lowest } \\
\text { risk is in homozygous E2 individuals. }\end{array}$ & $\begin{array}{l}{[48,65,} \\
76]\end{array}$ \\
\hline PICALM & $\mathrm{AD}$ & $\begin{array}{l}\text { rs3851179 } \\
\text { rs10792832 }\end{array}$ & $\begin{array}{l}\text { Encodes phosphatidylinositol-binding clathrin assembly } \\
\text { protein (PICALM), considered to be one of numerous } \\
\text { reproducible risk genes for LOAD. }\end{array}$ & [49] \\
\hline SPON1 & Dementia & rs2618516 & $\begin{array}{l}\text { Encodes the developmentally regulated protein F-spondin, } \\
\text { reported to be a putative ligand for the amyloid precursor } \\
\text { protein (APP). }\end{array}$ & {$[50]$} \\
\hline TCFLC2 & Diabetes & rs7903146 & $\begin{array}{l}\text { Associated with impaired insulin secretion and enhanced } \\
\text { hepatic glucose production. }\end{array}$ & {$[52]$} \\
\hline$A R I D 5 B$ & $\mathrm{AD}$ & rs2588969 & $\begin{array}{l}\text { Gene encodes a member of AT-rich interaction domain } \\
\text { (ARID) family of DNA binding proteins. The encoded } \\
\text { protein forms a histone H3K9Me } 2 \text { demethylase complex } \\
\text { with PHD finger protein } 2 \text { and regulates the transcription of } \\
\text { target genes involved in adipogenesis and liver } \\
\text { development. }\end{array}$ & {$[51]$} \\
\hline PILRA & $\mathrm{AD}$ & rs1859788 & $\begin{array}{l}\text { A cell surface inhibitory receptor that recognizes specific } \\
\text { O-glycosylated proteins and expressed on various innate } \\
\text { immune cell types including microglia }\end{array}$ & {$[53]$} \\
\hline CYP $3 A 5$ & $\begin{array}{l}\text { Salt retention } \\
\text { and } \\
\text { hypertension }\end{array}$ & rs776746 & $\begin{array}{l}\text { Cytochrome P450 (CYP) genes are abundant in animal, } \\
\text { plant, and bacterial genomes and have evolved to } \\
\text { metabolize a variety of diverse compounds. }\end{array}$ & {$[54]$} \\
\hline$P P A R G$ & Diabetes & rs1801282 & A nuclear hormone receptor that regulates adipogenesis & {$[55]$} \\
\hline BIN1 & $\mathrm{AD}$ & rs7561528 & $\begin{array}{l}\text { Also known as amphiphysin } 2 \text {, has recently been identified } \\
\text { as the most important LOAD risk locus }\end{array}$ & {$[20]$} \\
\hline$S C G 2$ & Hypertension & rs1017448 & Secretogranin II (SCG2) associates with hypertension & {$[56]$} \\
\hline CAPN10 & Diabetes & rs2975760 & $\begin{array}{l}\text { CAPN10 encodes a member of the calpain-like cysteine } \\
\text { protease family that regulates blood glucose levels. }\end{array}$ & {$[57]$} \\
\hline$A G T$ & $\begin{array}{c}\text { Sodium } \\
\text { retention }\end{array}$ & rs699 & Sodium homeostasis links with hypertension & {$[58]$} \\
\hline
\end{tabular}

770 Supplemental Table S1: Genes affecting cognitive functions in post-reproductive age exhibiting

771 disease-protective alleles uniquely in humans. The corresponding references for each of the genes

772 are mentioned in the table. 


\begin{tabular}{|c|c|c|c|c|c|c|c|c|}
\hline Gene & SNP ID & Allele & $\begin{array}{c}\text { Global } \\
\text { frequency }\end{array}$ & African & $\begin{array}{c}\text { East } \\
\text { Asian }\end{array}$ & European & $\begin{array}{l}\text { South } \\
\text { Asian }\end{array}$ & American \\
\hline \multirow{4}{*}{$C D 33$} & \multirow{2}{*}{ rs12459419 } & $\mathrm{C}$ & 0.789 & 0.949 & 0.814 & 0.69 & 0.84 & 0.52 \\
\hline & & $\mathbf{T}$ & 0.211 & 0.051 & 0.186 & 0.31 & 0.16 & 0.48 \\
\hline & \multirow{2}{*}{ rs3865444 } & $\mathrm{C}$ & 0.789 & 0.949 & 0.814 & 0.69 & 0.84 & 0.52 \\
\hline & & $\mathbf{A}$ & 0.211 & 0.051 & 0.186 & 0.31 & 0.16 & 0.48 \\
\hline \multirow{4}{*}{$A P O E$} & \multirow{2}{*}{ rs7412 } & $\mathrm{C}$ & 0.925 & 0.897 & 0.9 & 0.937 & 0.96 & 0.96 \\
\hline & & $\mathbf{T}$ & 0.075 & 0.103 & 0.1 & 0.063 & 0.04 & 0.04 \\
\hline & \multirow{2}{*}{ rs429358 } & $\mathbf{T}$ & 0.849 & 0.732 & 0.914 & 0.845 & 0.91 & 0.9 \\
\hline & & $\mathrm{C}$ & 0.151 & 0.268 & 0.086 & 0.155 & 0.09 & 0.1 \\
\hline \multirow{4}{*}{ PICALM } & \multirow{2}{*}{ rs3851179 } & $\mathbf{T}$ & 0.351 & 0.105 & 0.407 & 0.371 & 0.39 & 0.39 \\
\hline & & $\mathrm{C}$ & 0.685 & 0.895 & 0.593 & 0.629 & 0.61 & 0.61 \\
\hline & \multirow{2}{*}{ rs10792832 } & $\mathbf{A}$ & 0.313 & 0.094 & 0.409 & 0.372 & 0.4 & 0.39 \\
\hline & & $\mathrm{G}$ & 0.685 & 0.895 & 0.593 & 0.628 & 0.6 & 0.61 \\
\hline \multirow{2}{*}{ SPON1 } & \multirow{2}{*}{ rs2618516 } & $\mathbf{T}$ & 0.341 & 0.259 & 0.302 & 0.382 & 0.52 & 0.24 \\
\hline & & $\mathrm{C}$ & 0.659 & 0.741 & 0.698 & 0.618 & 0.48 & 0.76 \\
\hline \multirow{2}{*}{ TCFLC2 } & \multirow{2}{*}{ rs7903146 } & $\mathbf{C}$ & 0.772 & 0.74 & 0.977 & 0.683 & 0.7 & 0.77 \\
\hline & & $\mathrm{T}$ & 0.228 & 0.26 & 0.0023 & 0.317 & 0.3 & 0.23 \\
\hline \multirow{2}{*}{$A R I D 5 B$} & \multirow{2}{*}{ rs2588969 } & $\mathbf{C}$ & 0.532 & 0.472 & 0.482 & 0.641 & 0.63 & 0.42 \\
\hline & & $\mathrm{A}$ & 0.468 & 0.528 & 0.518 & 0.359 & 0.37 & 0.58 \\
\hline \multirow{2}{*}{ PILRA } & \multirow{2}{*}{ rs1859788 } & $\mathbf{A}$ & 0.341 & 0.102 & 0.612 & 0.321 & 0.29 & 0.5 \\
\hline & & $\mathrm{G}$ & 0.659 & 0.898 & 0.388 & 0.679 & 0.71 & 0.5 \\
\hline \multirow{2}{*}{ CYP3A5 } & \multirow{2}{*}{ rs776746 } & $\mathrm{T}$ & 0.379 & 0.82 & 0.287 & 0.05 & 0.33 & 0.2 \\
\hline & & $\mathbf{C}$ & 0.621 & 0.18 & 0.713 & 0.95 & 0.67 & 0.8 \\
\hline \multirow{2}{*}{$P P A R G$} & \multirow{2}{*}{ rs1801282 } & $\mathrm{C}$ & 0.93 & 0.995 & 0.974 & 0.88 & 0.88 & 0.88 \\
\hline & & $\mathbf{G}$ & 0.07 & 0.005 & 0.026 & 0.12 & 0.12 & 0.12 \\
\hline \multirow{2}{*}{ BIN1 } & \multirow{2}{*}{ rs7561528 } & $\mathbf{G}$ & 0.8 & 0.809 & 0.881 & 0.683 & 0.87 & 0.74 \\
\hline & & $\mathrm{A}$ & 0.2 & 0.191 & 0.119 & 0.317 & 0.13 & 0.26 \\
\hline \multirow{2}{*}{$S G C 2$} & \multirow{2}{*}{ rs1017448 } & $\mathbf{C}$ & 0.879 & 0.635 & 0.963 & 0.979 & 0.97 & 0.95 \\
\hline & & $\mathrm{T}$ & 0.121 & 0.365 & 0.037 & 0.021 & 0.03 & 0.05 \\
\hline \multirow{2}{*}{ CAPN10 } & \multirow{2}{*}{ rs2975760 } & $\mathbf{T}$ & 0.882 & 0.971 & 0.907 & 0.841 & 0.79 & 0.87 \\
\hline & & $\mathrm{C}$ & 0.118 & 0.029 & 0.093 & 0.159 & 0.21 & 0.13 \\
\hline \multirow{2}{*}{$A G T$} & rs600 & $\mathbf{A}$ & 0.295 & 0.097 & 0.147 & 0.588 & 0.36 & 0.36 \\
\hline & ISO99 & $\mathrm{G}$ & 0.705 & 0.903 & 0.853 & 0.412 & 0.64 & 0.64 \\
\hline
\end{tabular}

774 Supplemental Table S2: Analysis of Gene variants directly or indirectly affecting cognitive

775 function with their human population frequency. The global frequency of the SNPs identified in

776 Supplemental Table S1 was studied across different populations as indicated in the top of the 777 columns. 


\begin{tabular}{|c|c|c|c|}
\hline $\begin{array}{l}\text { Amino } \\
\text { acid } \\
\text { position }\end{array}$ & $\begin{array}{c}\text { Human } \\
\text { CD33(M)_EK_Fc } \\
\text { Variant }\end{array}$ & $\begin{array}{c}\text { Chimpanzee } \\
\text { CD33(M)_EK_Fc } \\
\text { Variant }\end{array}$ & $\begin{array}{c}\text { Mutagenesis Primer } \\
\text { Pairs_Forward/Reverse_5' }>3 \text {, }\end{array}$ \\
\hline 20 & N20K & - & $\begin{array}{l}\text { TGGATCCAAAaTTCTGGCTGCAAGTGCAGG } \\
\text { TAGCCAGGGCCCCTGCCC }\end{array}$ \\
\hline 21 & F21I & - & $\begin{array}{c}\text { GGATCCAAATaTCTGGCTGCAAGTGCAG } \\
\text { ATAGCCAGGGCCCCTGCC }\end{array}$ \\
\hline 22 & W22R & - & $\begin{array}{c}\text { TCCAAATTTCcGGCTGCAAGTGCAGG } \\
\text { TCCATAGCCAGGGCCCCT }\end{array}$ \\
\hline 65 & $\mathrm{~A} 65 \mathrm{P}$ & - & $\begin{array}{c}\text { CCGGGAAGGAcCCATTATATC } \\
\text { AACCAGTAACCATGAACTG }\end{array}$ \\
\hline 66 & I66F & - & $\begin{array}{l}\text { GGAAGGAGCCtTTATATCCAGG } \\
\text { CGGAACCAGTAACCATGAAC }\end{array}$ \\
\hline 67 & I67V & - & $\begin{array}{l}\text { AGGAGCCATTgTATCCAGGGAC } \\
\text { TCCCGGAACCAGTAACCA }\end{array}$ \\
\hline 69 & R69G & - & $\begin{array}{l}\text { CATTATATCCgGGGACTCTCCAGTG } \\
\text { GCTCCTTCCCGGAACCAG }\end{array}$ \\
\hline 78 & L78P & - & $\begin{array}{l}\text { ACAAACAAGCcAGATCAAGAAGTACAGGAG } \\
\text { GGCCACTGGAGAGTCCCT }\end{array}$ \\
\hline 96 & P96L & - & $\begin{array}{l}\text { CTTGGGGATCtCAGTAGGAACAAC } \\
\text { GAGGCGGAATCTGCCCTG }\end{array}$ \\
\hline 148 & L148V & - & $\begin{array}{l}\text { GCCCAAAATCgTCATCCCTGG } \\
\text { CTGTGGGTCAAGTCTGTC }\end{array}$ \\
\hline 152 & $\mathrm{~T} 152 \mathrm{~A}$ & - & $\begin{array}{l}\text { CATCCCTGGCgCTCTAGAACC } \\
\text { AGGATTTTGGGCCTGTGG }\end{array}$ \\
\hline 154 & E154D & - & $\begin{array}{l}\text { GCACTCTAGAtCCCGGCCACT } \\
\text { CAGGGATGAGGATTTTGGG }\end{array}$ \\
\hline 21 & - & $\mathrm{I} 21 \mathrm{~F}$ & $\begin{array}{l}\text { GGATCCAAAAtTCCGGCTGCAAGTG } \\
\text { ATAGCCAGGGCCCCTGTG }\end{array}$ \\
\hline 20 & - & K20N & $\begin{array}{c}\text { TGGATCCAAAtATCCGGCTGCAAGTGC } \\
\text { TAGCCAGGGCCCCTGTGG }\end{array}$ \\
\hline
\end{tabular}

779 Supplemental Table S3: List of the mutagenesis primers used in the study to generate the CD33

780 mutants. Lowercase letters correspond to base change. 
781 Supplemental File S1: List of the glycans used for the sialoglycan microarray. The complete

782 list of the chemoenzymatically synthesized glycans used to determine the binding profile of

783 different CD33 proteins are presented. The binding intensity of the different proteins (indicated on

784 the top of the columns) towards the corresponding glycan are shown in the heatmap (same heatmap

785 as in Figure 3). The red indicates maximum, and blue indicates minimum binding. $\mathrm{R}=$

786 propylamine linker present in the underlying glycan structure. Gal = galactose, GalNAc $=N-$

787 acetylgalactosamine, Glc $=$ glucose, GlcNAc $=N$-acetyl glucosamine, Fuc $=$ L-fucose. The linkage

788 between the monosaccharides is indicated as $\alpha$ - or $\beta$ - with numbers. 
1. Hawkes K., O'Connell J.F., Jones N.G., Alvarez H., and Charnov E.L. (1998).

Grandmothering, menopause, and the evolution of human life histories. Proc Natl Acad Sci U S A. 95, 1336-1339.

2. Hawkes K. (2004). Human longevity: the grandmother effect. Nature. 428, 128-129.

3. Hawkes K. (2010). Colloquium paper: how grandmother effects plus individual variation in frailty shape fertility and mortality: guidance from human-chimpanzee comparisons. Proc Natl Acad Sci U S A. 107 Suppl 2, 8977-8984.

4. Johnstone R.A., and Cant M.A. (2010). The evolution of menopause in cetaceans and humans: the role of demography. Proc Biol Sci. 277, 3765-3771.

5. Cant M.A., and Croft D.P. (2019). Life-History Evolution: Grandmothering in Space and Time. Curr Biol. 29, R215-R218.

6. Khan N., Kim S.K., Gagneux P., Dugan L.L., and Varki A. (2020). Maximum reproductive lifespan correlates with CD33rSIGLEC gene number: Implications for NADPH oxidasederived reactive oxygen species in aging. FASEB J. 34, 1928-1938.

7. Byars S.G., and Voskarides K. (2020). Antagonistic Pleiotropy in Human Disease. J Mol Evol. 88, 12-25.

8. Williams G.C. (1957). Pleiotropy, Natural Selection, and the Evolution of Senescence. Evolution. 11, 398-411.

9. Schwarz F., Springer S.A., Altheide T.K., Varki N.M., Gagneux P., and Varki A. (2016). Human-specific derived alleles of CD33 and other genes protect against postreproductive cognitive decline. Proc Natl Acad Sci U S A. 113, 74-79.

10. Hawkes K. (2016). Genomic evidence for the evolution of human postmenopausal longevity. Proc Natl Acad Sci U S A. 113, 17-18.

11. Läubli H., and Varki A. (2020). Sialic acid-binding immunoglobulin-like lectins (Siglecs) detect self-associated molecular patterns to regulate immune responses. Cell Mol Life Sci. 77, 593-605.

12. Siddiqui S.S., Springer S.A., Verhagen A., Sundaramurthy V., Alisson-Silva F., Jiang W., Ghosh P., and Varki A. (2017). The Alzheimer's disease-protective CD33 splice variant mediates adaptive loss of function via diversion to an intracellular pool. J Biol Chem. 292, 15312-15320.

13. Freeman S.D., Kelm S., Barber E.K., and Crocker P.R. (1995). Characterization of CD33 as a new member of the sialoadhesin family of cellular interaction molecules. Blood. 85, 20052012.

14. Bornhöfft K.F., Goldammer T., Rebl A., and Galuska S.P. (2018). Siglecs: A journey through the evolution of sialic acid-binding immunoglobulin-type lectins. Dev Comp Immunol. 86, 219-231.

15. Hernandez-Caselles T., Martinez-Esparza M., Perez-Oliva A.B., Quintanilla-Cecconi A.M., Garcia-Alonso A., Alvarez-Lopez D.M., and Garcia-Penarrubia P. (2006). A study of CD33 (SIGLEC-3) antigen expression and function on activated human T and NK cells: two isoforms of CD33 are generated by alternative splicing. J Leukoc Biol. 79, 46-58.

16. Malik M., Simpson J.F., Parikh I., Wilfred B.R., Fardo D.W., Nelson P.T., and Estus S. (2013). CD33 Alzheimer's risk-altering polymorphism, CD33 expression, and exon 2 splicing. J Neurosci. 33, 13320-13325. 
17. Saha S., Siddiqui S.S., Khan N., Verhagen A., Jiang W., Springer S., Ghosh P., and Varki A. (2019). Controversies about the subcellular localization and mechanisms of action of the Alzheimer's disease-protective CD33 splice variant. Acta Neuropathol. 138, 671-672.

18. Lamba J.K., Pounds S., Cao X., Downing J.R., Campana D., Ribeiro R.C., Pui C.H., and Rubnitz J.E. (2009). Coding polymorphisms in CD33 and response to gemtuzumab ozogamicin in pediatric patients with AML: a pilot study. Leukemia. 23, 402-404.

19. Bradshaw E.M., Chibnik L.B., Keenan B.T., Ottoboni L., Raj T., Tang A., Rosenkrantz L.L., Imboywa S., Lee M., Von Korff A. et al. (2013). CD33 Alzheimer's disease locus: altered monocyte function and amyloid biology. Nat Neurosci. 16, 848-850.

20. Naj A.C., Jun G., Beecham G.W., Wang L.S., Vardarajan B.N., Buros J., Gallins P.J., Buxbaum J.D., Jarvik G.P., Crane P.K. et al. (2011). Common variants at MS4A4/MS4A6E, CD2AP, CD33 and EPHA1 are associated with late-onset Alzheimer's disease. Nat Genet. $43,436-441$.

21. Hollingworth P., Harold D., Sims R., Gerrish A., Lambert J.C., Carrasquillo M.M., Abraham R., Hamshere M.L., Pahwa J.S., Moskvina V. et al. (2011). Common variants at ABCA7, MS4A6A/MS4A4E, EPHA1, CD33 and CD2AP are associated with Alzheimer's disease. Nat Genet. 43, 429-435.

22. Brinkman-Van der Linden E.C., Angata T., Reynolds S.A., Powell L.D., Hedrick S.M., and Varki A. (2003). CD33/Siglec-3 binding specificity, expression pattern, and consequences of gene deletion in mice. Mol Cell Biol. 23, 4199-4206.

23. Edler M.K., Sherwood C.C., Meindl R.S., Hopkins W.D., Ely J.J., Erwin J.M., Mufson E.J., Hof P.R., and Raghanti M.A. (2017). Aged chimpanzees exhibit pathologic hallmarks of Alzheimer's disease. Neurobiol Aging. 59, 107-120.

24. Edler M.K., Munger E.L., Meindl R.S., Hopkins W.D., Ely J.J., Erwin J.M., Mufson E.J., Hof P.R., Sherwood C.C., and Raghanti M.A. (2020). Neuron loss associated with age but not Alzheimer's disease pathology in the chimpanzee brain. Philos Trans R Soc Lond B Biol Sci. 375, 20190619.

25. Padler-Karavani V., Hurtado-Ziola N., Chang Y.C., Sonnenburg J.L., Ronaghy A., Yu H., Verhagen A., Nizet V., Chen X., Varki N. et al. (2014). Rapid evolution of binding specificities and expression patterns of inhibitory CD33-related Siglecs in primates. FASEB J. 28, 1280-1293.

26. Varki A. (2011). Since there are PAMPs and DAMPs, there must be SAMPs? Glycan "selfassociated molecular patterns" dampen innate immunity, but pathogens can mimic them. Glycobiology. 21, 1121-1124.

27. Varki A., and Gagneux P. (2012). Multifarious roles of sialic acids in immunity. Ann N Y Acad Sci. 1253, 16-36.

28. Tortorici M.A., Walls A.C., Lang Y., Wang C., Li Z., Koerhuis D., Boons G.J., Bosch B.J., Rey F.A., de Groot R.J. et al. (2019). Structural basis for human coronavirus attachment to sialic acid receptors. Nat Struct Mol Biol. 26, 481-489.

29. Tsai T.Y., Huang M.T., Sung P.S., Peng C.Y., Tao M.H., Yang H.I., Chang W.C., Yang A.S., Yu C.M., Lin Y.P. et al. (2021). SIGLEC-3 (CD33) serves as an immune checkpoint receptor for HBV infection. J Clin Invest. 131, 141965.

30. Varki A., and Angata T. (2006). Siglecs--the major subfamily of I-type lectins. Glycobiology. 16, 1R-27R. 
31. Consortium G.P., Auton A., Brooks L.D., Durbin R.M., Garrison E.P., Kang H.M., Korbel J.O., Marchini J.L., McCarthy S., McVean G.A. et al. (2015). A global reference for human genetic variation. Nature. 526, 68-74.

32. Prado-Martinez J., Sudmant P.H., Kidd J.M., Li H., Kelley J.L., Lorente-Galdos B., Veeramah K.R., Woerner A.E., O’Connor T.D., Santpere G. et al. (2013). Great ape genetic diversity and population history. Nature. 499, 471-475.

33. Xue Y., Prado-Martinez J., Sudmant P.H., Narasimhan V., Ayub Q., Szpak M., Frandsen P., Chen Y., Yngvadottir B., Cooper D.N. et al. (2015). Mountain gorilla genomes reveal the impact of long-term population decline and inbreeding. Science. 348, 242-245.

34. de Manuel M., Kuhlwilm M., Frandsen P., Sousa V.C., Desai T., Prado-Martinez J., Hernandez-Rodriguez J., Dupanloup I., Lao O., Hallast P. et al. (2016). Chimpanzee genomic diversity reveals ancient admixture with bonobos. Science. 354, 477-481.

35. Green R.E., Krause J., Briggs A.W., Maricic T., Stenzel U., Kircher M., Patterson N., Li H., Zhai W., Fritz M.H. et al. (2010). A draft sequence of the Neandertal genome. Science. 328, 710-722.

36. Prufer K., Racimo F., Patterson N., Jay F., Sankararaman S., Sawyer S., Heinze A., Renaud G., Sudmant P.H., de Filippo C. et al. (2014). The complete genome sequence of a Neanderthal from the Altai Mountains. Nature. 505, 43-49.

37. Edwards J.L., and Apicella M.A. (2004). The molecular mechanisms used by Neisseria gonorrhoeae to initiate infection differ between men and women. Clin Microbiol Rev. 17, 965-981.

38. Landig C.S., Hazel A., Kellman B.P., Fong J.J., Schwarz F., Agarwal S., Varki N., Massari P., Lewis N.E., Ram S. et al. (2019). Evolution of the exclusively human pathogen Neisseria gonorrhoeae: Human-specific engagement of immunoregulatory Siglecs. Evol Appl. 12, 337-349.

39. Apicella M.A., Mandrell R.E., Shero M., Wilson M.E., Griffiss J.M., Brooks G.F., Lammel C., Breen J.F., and Rice P.A. (1990). Modification by sialic acid of Neisseria gonorrhoeae lipooligosaccharide epitope expression in human urethral exudates: an immunoelectron microscopic analysis. J Infect Dis. 162, 506-512.

40. Parsons N.J., Patel P.V., Tan E.L., Andrade J.R., Nairn C.A., Goldner M., Cole J.A., and Smith H. (1988). Cytidine 5'-monophospho-N-acetyl neuraminic acid and a low molecular weight factor from human blood cells induce lipopolysaccharide alteration in gonococci when conferring resistance to killing by human serum. Microb Pathog. 5, 303-309.

41. Caugant D.A., and Brynildsrud O.B. (2020). Neisseria meningitidis: using genomics to understand diversity, evolution and pathogenesis. Nat Rev Microbiol. 18, 84-96.

42. Seifert H.S. (2019). Location, Location, Location-Commensalism, Damage and Evolution of the Pathogenic Neisseria. J Mol Biol. 431, 3010-3014.

43. Carlin A.F., Lewis A.L., Varki A., and Nizet V. (2007). Group B streptococcal capsular sialic acids interact with siglecs (immunoglobulin-like lectins) on human leukocytes. J Bacteriol. 189, 1231-1237.

44. Fong J.J., Tsai C.M., Saha S., Nizet V., Varki A., and Bui J.D. (2018). Siglec-7 engagement by GBS $\beta$-protein suppresses pyroptotic cell death of natural killer cells. Proc Natl Acad Sci U S A. 115, 10410-10415.

45. Munshi M.N. (2017). Cognitive Dysfunction in Older Adults With Diabetes: What a Clinician Needs to Know. Diabetes Care. 40, 461-467. 
964

965

966

967
46. Peila R., Rodriguez B.L., Launer L.J., and Honolulu-Asia A.S. (2002). Type 2 diabetes, APOE gene, and the risk for dementia and related pathologies: The Honolulu-Asia Aging Study. Diabetes. 51, 1256-1262.

47. Raj T., Ryan K.J., Replogle J.M., Chibnik L.B., Rosenkrantz L., Tang A., Rothamel K., Stranger B.E., Bennett D.A., Evans D.A. et al. (2014). CD33: increased inclusion of exon 2 implicates the Ig V-set domain in Alzheimer's disease susceptibility. Hum Mol Genet.

48. Finch C.E., and Sapolsky R.M. (1999). The evolution of Alzheimer disease, the reproductive schedule, and apoE isoforms. Neurobiol Aging. 20, 407-428.

49. Xu W., Tan L., and Yu J.T. (2015). The Role of PICALM in Alzheimer's Disease. Mol Neurobiol. 52, 399-413.

50. Liu Z., Dai X., Tao W., Liu H., Li H., Yang C., Zhang J., Li X., Chen Y., Ma C. et al. (2018). APOE influences working memory in non-demented elderly through an interaction with SPON1 rs2618516. Hum Brain Mapp. 39, 2859-2867.

51. Carrasquillo M.M., Belbin O., Hunter T.A., Ma L., Bisceglio G.D., Zou F., Crook J.E., Pankratz V.S., Sando S.B., Aasly J.O. et al. (2011). Replication of EPHA1 and CD33 associations with late-onset Alzheimer's disease: a multi-centre case-control study. Mol Neurodegener. 6, 54.

52. Helgason A., Pálsson S., Thorleifsson G., Grant S.F., Emilsson V., Gunnarsdottir S., Adeyemo A., Chen Y., Chen G., Reynisdottir I. et al. (2007). Refining the impact of TCF7L2 gene variants on type 2 diabetes and adaptive evolution. Nat Genet. 39, 218-225.

53. Rathore N., Ramani S.R., Pantua H., Payandeh J., Bhangale T., Wuster A., Kapoor M., Sun Y., Kapadia S.B., Gonzalez L. et al. (2018). Paired Immunoglobulin-like Type 2 Receptor Alpha G78R variant alters ligand binding and confers protection to Alzheimer's disease. PLoS Genet. 14, e1007427.

54. Thompson E.E., Kuttab-Boulos H., Witonsky D., Yang L., Roe B.A., and Di Rienzo A. (2004). CYP3A variation and the evolution of salt-sensitivity variants. Am J Hum Genet. 75, 1059-1069.

55. Altshuler D., Hirschhorn J.N., Klannemark M., Lindgren C.M., Vohl M.C., Nemesh J., Lane C.R., Schaffner S.F., Bolk S., Brewer C. et al. (2000). The common PPARgamma Pro12Ala polymorphism is associated with decreased risk of type 2 diabetes. Nat Genet. 26, 76-80.

56. Wen G., Wessel J., Zhou W., Ehret G.B., Rao F., Stridsberg M., Mahata S.K., Gent P.M., Das M., Cooper R.S. et al. (2007). An ancestral variant of Secretogranin II confers regulation by PHOX2 transcription factors and association with hypertension. Hum Mol Genet. 16, $1752-1764$.

57. Vander Molen J., Frisse L.M., Fullerton S.M., Qian Y., Del Bosque-Plata L., Hudson R.R., and Di Rienzo A. (2005). Population genetics of CAPN10 and GPR35: implications for the evolution of type 2 diabetes variants. Am J Hum Genet. 76, 548-560.

58. Nakajima T., Wooding S., Sakagami T., Emi M., Tokunaga K., Tamiya G., Ishigami T., Umemura S., Munkhbat B., Jin F. et al. (2004). Natural selection and population history in the human angiotensinogen gene (AGT): 736 complete AGT sequences in chromosomes from around the world. Am J Hum Genet. 74, 898-916.

59. Iadecola C. (2013). The pathobiology of vascular dementia. Neuron. 80, 844-866.

60. Reich D., Green R.E., Kircher M., Krause J., Patterson N., Durand E.Y., Viola B., Briggs A.W., Stenzel U., Johnson P.L. et al. (2010). Genetic history of an archaic hominin group from Denisova Cave in Siberia. Nature. 468, 1053-1060. 
1003

1004

1005

1006

1007

1008

1009

1010

1011

1012

1013

61. Meyer M., Kircher M., Gansauge M.T., Li H., Racimo F., Mallick S., Schraiber J.G., Jay F., Prufer K., de Filippo C. et al. (2012). A high-coverage genome sequence from an archaic Denisovan individual. Science. 338, 222-226.

62. Khan N., de Manuel M., Peyregne S., Do R., Prufer K., Marques-Bonet T., Varki N., Gagneux P., and Varki A. (2020). Multiple Genomic Events Altering Hominin SIGLEC Biology and Innate Immunity Predated the Common Ancestor of Humans and Archaic Hominins. Genome Biol Evol. 12, 1040-1050.

63. Mendez F.L., Poznik G.D., Castellano S., and Bustamante C.D. (2016). The Divergence of Neandertal and Modern Human Y Chromosomes. Am J Hum Genet. 98, 728-734.

64. Moon J.M., Aronoff D.M., Capra J.A., Abbot P., and Rokas A. (2018). Examination of Signatures of Recent Positive Selection on Genes Involved in Human Sialic Acid Biology. G3 (Bethesda). 8, 1315-1325.

65. Reiman E.M., Arboleda-Velasquez J.F., Quiroz Y.T., Huentelman M.J., Beach T.G., Caselli R.J., Chen Y., Su Y., Myers A.J., Hardy J. et al. (2020). Exceptionally low likelihood of Alzheimer's dementia in APOE2 homozygotes from a 5,000-person neuropathological study. Nat Commun. 11, 667.

66. Oriá R.B., Patrick P.D., Zhang H., Lorntz B., de Castro Costa C.M., Brito G.A., Barrett L.J., Lima A.A., and Guerrant R.L. (2005). APOE4 protects the cognitive development in children with heavy diarrhea burdens in Northeast Brazil. Pediatr Res. 57, 310-316.

67. Angata T., and Varki A. (2000). Cloning, characterization, and phylogenetic analysis of siglec-9, a new member of the CD33-related group of siglecs. Evidence for co-evolution with sialic acid synthesis pathways. J Biol Chem. 275, 22127-22135.

68. Angata T., Kerr S.C., Greaves D.R., Varki N.M., Crocker P.R., and Varki A. (2002). Cloning and characterization of human Siglec-11. A recently evolved signaling molecule that can interact with SHP-1 and SHP-2 and is expressed by tissue macrophages, including brain microglia. J Biol Chem. 277, 24466-24474.

69. Kumar S., Stecher G., and Tamura K. (2016). MEGA7: Molecular Evolutionary Genetics Analysis Version 7.0 for Bigger Datasets. Mol Biol Evol. 33, 1870-1874.

70. Krieger E., and Vriend G. (2014). YASARA View - molecular graphics for all devices - from smartphones to workstations. Bioinformatics. 30, 2981-2982.

71. Krieger E., and Vriend G. (2015). New ways to boost molecular dynamics simulations. J Comput Chem. 36, 996-1007.

72. Humphrey W., Dalke A., and Schulten K. (1996). VMD: visual molecular dynamics. J Mol Graph. 14, 33-8, 27.

73. Meng C., Sasmal A., Zhang Y., Gao T., Liu C.C., Khan N., Varki A., Wang F., and Cao H. (2018). Chemoenzymatic Assembly of Mammalian O-Mannose Glycans. Angew Chem Int Ed Engl. 57, 9003-9007.

74. Lu N., Ye J., Cheng J., Sasmal A., Liu C.C., Yao W., Yan J., Khan N., Yi W., Varki A. et al. (2019). Redox-Controlled Site-Specific a2-6-Sialylation. J Am Chem Soc. 141, 4547-4552.

75. Ji Y., Sasmal A., Li W., Oh L., Srivastava S., Hargett A.A., Wasik B.R., Yu H., Diaz S., Choudhury B. et al. (2021). Reversible O-Acetyl Migration within the Sialic Acid Side Chain and Its Influence on Protein Recognition. ACS Chem Biol. 16, 1951-1960.

76. Fullerton S.M., Clark A.G., Weiss K.M., Nickerson D.A., Taylor S.L., Stengârd J.H., Salomaa V., Vartiainen E., Perola M., Boerwinkle E. et al. (2000). Apolipoprotein E variation at the sequence haplotype level: implications for the origin and maintenance of a major human polymorphism. Am J Hum Genet. 67, 881-900. 